\title{
TINC - a method to dissect transcriptional complexes at single locus resolution - reveals novel Nanog regulators in mouse embryonic stem cells
}

Knaupp AS, ${ }^{1,2,3}$ Mohenska M, ${ }^{1,2,3}$ Larcombe MR,,${ }^{1,2,3}$ Ford E, ${ }^{4,5}$ Lim SM,,${ }^{1,2,3}$ Wong K,,${ }^{1,2,3}$ Chen J, ${ }^{1,2,3}$ Firas J, ${ }^{1,2,3}$ Huang C, ${ }^{6}$ Liu X, ${ }^{1,2,3}$ Nguyen T, ${ }^{4,5}$ Sun YBY,,${ }^{1,2,3}$ Holmes ML, ${ }^{1,2,3}$ Tripathi P, ${ }^{1,2}$ Rossello FJ, ${ }^{1,2,3}$ Schröder J, ${ }^{1,2,3}$ Nefzger CM, ${ }^{1,2,3}$ Das PP, ${ }^{1,2}$ Haigh JJ, ${ }^{7,8,9}$ Lister R, Schittenhelm RB, ${ }^{6}$ and Polo $\mathrm{JM}^{1,2,3, *}$

${ }^{1}$ Department of Anatomy and Developmental Biology, Monash University, VIC 3800, Australia

${ }^{2}$ Development and Stem Cells Program, Monash Biomedicine Discovery Institute, VIC 3800, Australia

${ }^{3}$ Australian Regenerative Medicine Institute, Monash University, VIC 3800, Australia

${ }^{4}$ Australian Research Council Centre of Excellence in Plant Energy Biology, School of Molecular Sciences, The University of Western Australia, WA 6009, Australia

${ }^{5}$ Harry Perkins Institute of Medical Research, WA 6009, Australia

${ }^{6}$ Department of Biochemistry and Molecular Biology, Monash University, VIC 3800, Australia ${ }^{7}$ Australian Centre for Blood Diseases, Monash University, VIC 3004, Australia

${ }^{8}$ Department of Pharmacology and Therapeutics, Rady Faculty of Health Sciences, University of Manitoba, Winnipeg, Manitoba, Canada

${ }^{9}$ Research Institute in Oncology and Hematology, CancerCare Manitoba, Winnipeg, Manitoba, Canada

*Correspondence: jose.polo@monash.edu

\begin{abstract}
Cellular identity is ultimately controlled by transcription factors (TFs), which bind to specific regulatory elements (REs) within the genome to regulate gene expression and cell fate changes. While recent advances in genome-wide epigenetic profiling techniques have significantly increased our understanding of which REs are utilized in which cell type, it remains largely unknown which TFs and cofactors interact with these REs to modulate gene expression. A major hurdle in dissecting the whole composition of a multi-protein complex formed at a specific RE is the shortage of appropriate techniques. We have developed a novel method termed TALE-mediated Isolation of Nuclear Chromatin (TINC). TINC utilizes epitope-tagged TALEs to isolate a specific genomic region from the mammalian genome and includes a nuclei isolation and chromatin enrichment step for increased specificity. Upon crosslinking of the cells and isolation of the chromatin, the target region is purified based on affinity purification of the TALE and associated nucleic acid and protein molecules can be subjected to further analyses. A key TF in the pluripotency network and therefore in embryonic stem cells (ESCs) is NANOG. It is currently not fully understood how Nanog expression is regulated and consequently it remains unclear how the ESC state is maintained. Using TINC we dissected the protein complex formed at the Nanog promoter in mouse ESCs and identified many known and numerous novel factors.
\end{abstract}




\section{Introduction}

Pluripotent stem cells (PSCs) carry immense therapeutic potential due to their ability to give rise to any cell type of the body and their capacity to self-renew indefinitely. The most common in vitro models of PSCs are embryonic stem cells (ESCs), which are obtained from the inner cell mass (ICM) of the blastocyst (Martin 1981; Kaufman et al. 1983) and induced pluripotent stem cells (iPSCs), which can be derived from somatic cells through overexpression of the four transcription factors (TFs) OCT4, SOX2, KLF4 and C-MYC (OSKM) (Takahashi and Yamanaka 2006). Besides OSKM, the homeobox TF NANOG has been shown to be involved in pluripotency maintenance of the ICM and of ESCs/iPSCs (Mitsui et al. 2003; Jose Silva et al. 2009). Together with OCT4 and SOX2, NANOG forms the core transcriptional regulatory circuitry that drives the ESC state (Boyer et al. 2005). These three TFs co-occupy many targets and collaborate to activate the expression of self-renewal and pluripotency genes including themselves and to suppress the expression of genes that promote differentiation (Loh et al. 2006; Boyer et al. 2005; Chen et al. 2008; Marson et al. 2008; Kim et al. 2008). Interestingly, out of the three core pluripotency TFs only NANOG seems to have the capacity to mediate LIF/STAT3-independent self-renewal of ESCs (Mitsui et al. 2003; Chambers et al. 2003).

Besides pluripotency maintenance, NANOG plays an important role during cellular reprogramming (José Silva et al. 2006; Jose Silva et al. 2009; Yu et al. 2007). There is evidence, however, that somatic cells can be reprogrammed in the absence of NANOG, albeit at much lower efficiency (Carter et al. 2014). Additionally, there is evidence that ESCs can be maintained upon genetic deletion of Nanog although the cells are prone to differentiation (Chambers et al. 2007). This observation, together with work revealing that Nanog is expressed in a heterogeneous manner in ESCs (Chambers et al. 2007; Kalmar et al. 2009; Kumar et al. 2014; Abranches et al. 2014), suggests that the pluripotency network can cope with variable levels of NANOG. Fluctuations in NANOG levels seem to allow ESCs to regulate lineage commitment, with high NANOG levels impeding and low levels promoting response to differentiation cues (Kalmar et al. 2009; Abranches et al. 2014; Chambers et al. 2003). How the expression of Nanog is regulated in ESCs remains, however, still not fully understood.

As any given gene, the expression of Nanog is controlled by TFs, which bind to specific regulatory elements (REs) and recruit other factors such as TFs, cofactors and epigenetic regulators to modulate gene expression. There are at least two major REs controlling Nanog expression in ESCs: one in the promoter region and an enhancer approximately $5 \mathrm{~kb}$ upstream of the TSS that loops to interact with the Nanog promoter (Kagey et al. 2010; Apostolou et al. 2013). NANOG has been shown to bind to both of these REs (Chen et al. 2008; Kim et al. 2008), and this NANOG activity has been associated with auto-activation (Q. Wu et al. 2006) as well as auto-repression (Fidalgo et al. 2012; Navarro et al. 2012). These positive and negative Nanog autoregulatory feedback loops are linked to NANOG interacting with different TFs. For example, SALL4 has been shown to physically associate with NANOG to activate Nanog expression (Q. Wu et al. 2006), whereas Nanog auto-repression has been shown to depend on its interaction with ZFP281, which in turn recruits the NuRD repressor complex (Fidalgo et al. 2012). Furthermore, positive transcriptional regulation of Nanog has been associated with binding of OCT4 and SOX2 to the Nanog promoter (Rodda et al. 2005), whereas Nanog auto-repression has been shown to be independent of OCT4 and SOX2 
(Navarro et al. 2012). This suggests that fine-tuning of Nanog expression levels might rely on differences in TF and cofactor interactions. However, while some of the proteins that interact with these RE are known, the full characterization of the complexes formed at any of these REs remains largely elusive.

One major reason for this lack of knowledge is that dissecting the whole molecular composition of a regulatory complex formed at a specific RE is very difficult to achieve. Chromatin immunoprecipitations (ChIPs) have been invaluable to study the genome-wide DNA binding events of proteins of interest, however, they require a priori knowledge in order to select a candidate protein, antibodies appropriate for this application and they allow the interrogation of only one protein at the time (reviewed in (Furey 2012; Collas 2010)). Consequently, being able to simultaneously analyze the entire set of proteins that interact at a specific RE would be advantageous, however, this is extremely challenging for two main reasons: 1) a very low abundant genomic region (e.g. two copies per cell) has to be specifically targeted and 2) the interacting proteins have to be enriched efficiently to be detected. Unlike DNA and RNA molecules, which can be easily amplified (e.g. PCR), proteins have to be interrogated without further amplification, which poses a significant challenge for low abundant RE-associated proteins. High-resolution liquid chromatography tandem mass spectrometry (LC-MS/MS) is the method of choice for such analyses. Hence, the number of proteins, which can be identified and quantified at a specific genomic locus, is primarily governed by the intrinsic properties of the mass spectrometer such as mass accuracy, resolving power and sensitivity, which in turn dictate the lower limit of detection and quantification for individual species (typically in the femtomole-on-column range).

Several groups have set out to overcome these limitations by developing various locusspecific isolation or proximity labelling methods in combination with mass spectrometry. These include nucleic acid hybridization-based approaches (Ide and Dejardin 2015; Déjardin and Kingston 2009; Antão et al. 2012; Kennedy-Darling et al. 2014) and approaches that utilize DNA-binding proteins including LexA (Byrum et al. 2012; Fujita and Fujii 2011), TetR (Pourfarzad et al. 2013), Cas9 (Waldrip et al. 2014; Fujita and Fujii 2013; X. Liu et al. 2017; Gao et al. 2018; Schmidtmann et al. 2016; Tsui et al. 2018; Qiu et al. 2019) or TALE proteins (Fujita et al. 2013; Byrum, Taverna, and Tackett 2013; Fang et al. 2018). Although nucleic acid hybridization-based approaches pioneered the field, they require intensive probe-specific optimizations (Ide and Dejardin 2015; Déjardin and Kingston 2009; Antão et al. 2012), which may account for why this approach has not yet been widely adopted nor translated to single copy elements in mammalian cells. To target such challenging genomic regions in the mammalian genome, TALE (Fang et al. 2018) and CRISPR/Cas9 (Waldrip et al. 2014; Fujita and Fujii 2013; X. Liu et al. 2017; Gao et al. 2018; Schmidtmann et al. 2016; Tsui et al. 2018; Qiu et al. 2019) approaches are starting to emerge as the systems of choice as, unlike TetR and LexA proteins, they do not rely on genetic engineering of binding sites into the target sequence.

Here, we describe a novel epigenetic method that allows the isolation of a specific genomic region from the complex mammalian genome termed TALE-mediated Isolation of Nuclear Chromatin (TINC). TINC utilizes an epitope-tagged TALE in combination with a nuclei isolation and chromatin enrichment step based on Kustatscher et al., 2014 to further increase the signal-to-noise ratio. Even though the only other mammalian cell-validated TALEbased single locus isolation technique utilizes whole cell lysate (Fang et al. 2018) we chose to 
include these two additional purification steps (nuclei and chromatin) to reduce cytoplasmic and free nuclear protein contaminant contribution. Using TINC, we interrogated the protein complex formed at the Nanog promoter in mouse ESCs. We were able to not only detect many proteins previously shown to interact with this key pluripotency RE such as OCT4, SOX2 and NANOG (Chen et al. 2008; Kim et al. 2008) but more importantly also many novel candidates.

\section{Results}

\section{Development of TINC to target the Nanog promoter in mESCs}

In order to interrogate the protein complex formed at the Nanog promoter in ESCs, we developed TINC (Figure 1A); an epigenetic technique applicable to essentially any genomic region. In short, a construct driving the expression of an epitope tagged TALE (3xHA-tag) designed to bind to the sequence of interest is introduced into mammalian cells (e.g. via transfection/transduction). Upon confirmation that the TALE is expressed (e.g. via western blot/immunofluorescence), the cells are fixed with formaldehyde, nuclei isolated followed by chromatin isolation (Kustatscher et al. 2014) to enrich chromatin-associated proteins and to further decrease background noise. The chromatin is then sheared by sonication and the target region is isolated based on affinity purification of the TALE (e.g. utilizing anti-HA agarose). After elution and further purification, nucleic acids and proteins can then be analysed by appropriate techniques including quantitative polymerase chain reaction (qPCR) and LCMS/MS, respectively. This allows the assessment whether the genomic locus of interest has been isolated and which proteins are associated with this genomic locus.

To investigate the protein complex regulating the transcription of the master pluripotency regulator NANOG, TINC was utilized to isolate the Nanog promoter from mouse ESCs. A TALE was designed to bind upstream of the OCT4 and SOX2 binding sites (Rodda et al. 2005) in this key RE (Figure 1B) and a clonal ESC line stably expressing this TALE was created (Figure S1A). A negative control line, transfected with the empty vector, was also generated (Figure S1A). TALE-expressing ESCs were characterized by unaltered Nanog expression (Figure 1C) and importantly, ChIP of the TALE resulted in clear enrichment of its genomic target: the Nanog promoter (Figure 1D). Furthermore, the capacity to give rise to cells from all three germ layers in teratoma assays was unaffected (Figure S1B). Altogether, these results suggest that binding of the TALE to the Nanog promoter does not change expression of the target gene nor the pluripotency potential of the cells.

To determine the proteins that interact with the Nanog promoter in mouse ESCs, we performed TINC and the enriched proteins were analysed by LC-MS/MS (Figures 1E and S1C). The proteins that were also detected in the negative controls were considered as background (please see discussion) and consequently discarded from further analysis (Figure $\mathrm{S} 1 \mathrm{C})$. Some of these proteins were found to be enriched in the TALE samples in comparison to the negative controls (data not shown) suggesting they might also interact with the Nanog promoter, however, we decided to be stringent and rather increase the number of false negatives than of false positives. To further increase the accuracy of our results, we included an additional cut-off (Vermeulen and Déjardin 2020) and only utilized TINC replicates for further analysis that identified the three expected key pluripotency regulators OCT4, SOX2 and NANOG. Overlap of the proteins detected uniquely in the TALE samples in three TINC replicates revealed 280 candidates to be present in all three replicates (Figure 1E). Importantly, besides 
OCT4, SOX2 and NANOG these included many other proteins that had previously been shown to interact with the Nanog promoter in mouse ESCs such as BRD4 (W. Liu et al. 2014), DIDO1 (Y. Liu et al. 2014), DPF2 (Zhang et al. 2019), ESRRB (Chen et al. 2008), KLF4 (Chen et al. 2008; Kim et al. 2008), MYBL2 (also known as B-MYB) (Zhan et al. 2012), NR0B1 (also known as DAX1) (Kim et al. 2008), TET1 (H. Wu et al. 2011), TET2 (Sardina et al. 2018), TCFCP2L1 (also known as TFCP2L1) (Chen et al. 2008) and ZFP281 (Wang et al. 2008). To further confirm the validity of our TINC technique, we utilized the database ChIP-Atlas, which allows data mining and visualization of published chromatin immunoprecipitations (ChIP) sequencing data sets (Oki et al. 2018). ChIP-Atlas revealed binding of an additional eight proteins within this genomic region in ESCs: BRD2, CTR9, DPPA4, NELFA, RNF2, SUMO1, SUMO2 and ZFP57 (Figure 1F). Interestingly, these proteins seem to interact with three distinct sites: the Nanog promoter and/ or the $-5 \mathrm{~kb}$ enhancer or a region in between these two REs.

We also validated by ChIP-qPCR five novel candidates, which are upregulated during MEF reprogramming (Knaupp et al. 2017) but had previously not been shown to interact with the Nanog promoter: RCOR2 (REST corepressor 2), DNMT3L (DNA (cytosine-5)methyltransferase 3-like), NFATC2IP (NFATC2-interacting protein), WDR76 (WD repeatcontaining protein 76) and ZFP57 (zinc finger protein 57) (Figure 1G). We included ZFP57 for which the ChIP-Atlas data base suggested targeting of the Nanog promoter (Figure 1F) while the original publication did not call this binding site (Strogantsev et al. 2015). To overcome the lack of ChIP antibodies against most of these proteins, we expressed 3xHA-tagged versions of these candidates in ESCs as well as of SOX2 (Chen et al. 2008; Kim et al. 2008) and MYBL2 (Zhan et al. 2012) as positive controls. ChIP-qPCR confirmed enrichment of these proteins at the Nanog promoter, with enrichment values in between the two positive controls SOX2 and MYBL2, the latter of which showed only very poor enrichment (Figure 1G).

Altogether, this demonstrates that TINC is an appropriate technique for the interrogation of the protein complex formed at the Nanog promoter in ESCs, and hence any given RE in the mammalian genome, with the potential to discover hundreds of interacting proteins.

\section{Interrogation of the protein complex formed at the Nanog promoter using TINC}

The 280 proteins found to interact at the Nanog promoter were further characterized to unveil their potential roles at this RE. Unsurprisingly, protein class ontology analysis showed the strongest enrichment for nucleic acid binding proteins (Figure 2A). Additionally, protein modifying enzymes (e.g. non-receptor serine/threonine protein kinases, proteases, protein phosphatases and ubiquitin-protein ligases), gene-specific transcriptional regulators and chromatin/chromatin-binding or chromatin-regulatory proteins were found to be strongly enriched (Figure 2A). Importantly, analysis of the 57 transcription factors identified by TINC revealed that they were enriched for biological processes and KEGG pathways associated with stem cell maintenance and pluripotency (Figure 2B). Further characterization of all 91 epigenetic modifiers showed that besides TFs associated with transcriptional activation and repression, many were histone modification writers or their cofactors (Figure S2A). Only a minority of factors we identified were chromatin remodelling (co-) factors or DNA modifiers (e.g. mediating DNA hydroxymethylation). Consequently, it is unsurprising that while the 
molecular target of the TFs is DNA, the molecular target of the majority of the other epigenetic modifiers we identified at the Nanog promoter are histones (Figure S2A). These results suggest that various and different epigenetic modifiers are required to regulate Nanog expression and that they exert their roles at various levels including modifications of the DNA and histone landscape.

In order to gain further insight into the protein interaction network we conducted protein network analysis and k-means clustering of all proteins identified by TINC using STRING (Szklarczyk et al. 2019). This approach revealed nine distinct clusters associated with covalent chromatin modifications, mRNA splicing, ribosome biogenesis, nuclear division, DNA replication, CCT (chaperonin containing TCP1 complex), stem cell population maintenance, nucleosome disassembly and double-strand break repair (Figure 2C). This further supports the above-mentioned notion that the proteins regulating the transcription of Nanog are required to exhibit differing functions at the DNA, RNA and protein level.

Since NANOG is one of the master regulators of pluripotency, we next sought to determine whether other key regulators of the pluripotency network control the transcription of this gene. Comparison of the proteins we identified at the Nanog promoter with the previously defined pluripotency regulatory network (Nefzger et al. 2017; Xu et al. 2013, 2014) revealed a significant enrichment of 77 proteins including OCT4, SOX2, NANOG and TCFCP2L1 (Note: SALL4 was identified in two of the three TINC replicates) (Figure 2D). This indicates that over a tenth of the key regulators of the pluripotency network are required to control the transcription of Nanog. These 77 proteins are broadly classified as nucleic acid binding proteins, transcriptional regulators, chromatin-chromatin binding or regulatory proteins, chaperones and protein modifying enzymes (phosphatases, proteases, kinases and E3 ubiquitin ligases) (Figure S2B).

As mentioned previously, NANOG does not only play an important role during pluripotency maintenance but also in attaining the pluripotent state during reprogramming (José Silva et al. 2006; Jose Silva et al. 2009; Yu et al. 2007). We therefore determined the expression dynamics during reprogramming of the proteins associated with the Nanog transcriptional complex and hence, when the pluripotency-associated complex is potentially established during this cellular conversion (Figure 2E). Analysis of the expression of the 280 TINC proteins during reprogramming of mouse embryonic fibroblasts (MEFs) into iPSCs revealed that 192 of these proteins are upregulated during this cellular conversion (Figure 2E). This suggests that these 192 proteins are specific to pluripotency meanwhile the remaining 88 proteins play common roles across cell types. Nanog expression is maintained at low levels during reprogramming and only increases significantly between day 12 and the iPSC state (Figure S2C). In order to determine whether this change in Nanog expression might be due to a change in the expression of some of the proteins that regulate the expression of Nanog in the pluripotent state we correlated their expression to the expression of Nanog throughout the reprogramming process (Figures S2D and S2E). Interestingly, most of these proteins were upregulated at the later stages of reprogramming and the expression of most of these proteins was positively correlated with the expression of Nanog (Figure S2D). 16\% of these highly correlated genes are TFs, $10 \%$ are epigenetic modifiers and $51 \%$ are other proteins many of which are associated with RNA modification and splicing. Furthermore, the $25 \%$ most correlated genes are associated with chromatin organization, dedifferentiation, cell cycle and 
genetic imprinting development (Figure S2F). Intriguingly, all of the proteins that were negatively correlated with the expression of Nanog were transiently upregulated during the early stages of reprogramming (Figure S2E). These genes are mostly involved in mRNA processing and protein ubiquitination (Figure S2F). Overall, this indicates that many of the proteins identified at the Nanog promoter in the pluripotent state are upregulated only at the final stages of cellular reprogramming. Importantly, this coincides with the initiation of Nanog expression during this cellular conversion (Figure S2C).

\section{Discussion}

Here, we describe TALE-mediated Isolation of Nuclear Chromatin (TINC), a novel epigenetic technique that allows isolation of a single genomic locus from mammalian cells for further molecular interrogation. TINC utilizes epitope tagged TALE proteins, which have previously been shown to possess the capacity to enrich a single RE from mammalian cells (Fang et al. 2018). In order to minimize background contribution from cytoplasmic contaminants as well as from proteins specifically enriched by the TALE but without target locus-related functions (e.g. proteins that interact with the nascent TALE polypeptide chain during translation), TINC contains a nuclei isolation and a chromatin enrichment step (Kustatscher et al. 2014).

We used TINC to interrogate the regulatory complex formed at the Nanog promoter in ESCs and only considered proteins that were solely detected in the TALE samples but not the negative controls. We acknowledge that this approach might discard some proteins that are indeed part of the studied complex (e.g. proteins that are enriched in the TALE samples in comparison to the negative controls), that therefore become false negatives. Additionally, Jérôme Déjardin, who, together with Robert Kingston, published the first protocol for the isolation of specific genomic DNA segments (human telomeres), stated recently that extreme caution should be exerted when interpreting such locus specific data that did not recover expected proteins (Vermeulen and Déjardin 2020). In agreement with Vermeulen and Déjardin, 2020, we therefore utilized previously identified binders as positive controls (e.g. the master pluripotency regulators OCT4, SOX2 and NANOG) and excluded one TINC replicate that did not allow identification of SOX2. Furthermore, we only considered proteins identified in all three valid TINC replicates as bound to the Nanog promoter. This stringent approach allowed us to identify many previously published Nanog promoter interactors including the key pluripotency factors OCT4, SOX2 and NANOG (Chen et al. 2008; Kim et al. 2008) as well as many proteins that had previously not been shown to bind to this RE. However, even if we had taken a completely unbiased approach and had not excluded the fourth TINC replicate that was missing SOX2, we would have identified many known Nanog binders besides OCT4 and NANOG including BRD4, DIDO1, DPF2, KLF4, MYBL2, NR0B1, TET1, TET2 and TFCP2L1 and more than 100 novel candidates. This suggests that while known binders are very helpful for setting analysis cut-offs, TINC can also give significant insight into REs without prior knowledge on interacting proteins.

To support the validity of our TINC method we confirmed by conventional ChIP-qPCR the binding of five novel proteins: RCOR2, ZFP57, DNMT3L, NFATC2IP and WDR76 to the Nanog promoter. As mentioned above, we needed to tag these proteins since we could not source ChIP antibodies. It is possible that the epitope tag interfered with folding or binding of 
these TFs and that is why ChIP could not enrich MYBL2 well at this RE. However, the commercial antibody previously used to show MYBL2 binding to the Nanog promoter (Zhan et al. 2012) has been discontinued. On that note, the authors of this study tested five commercial antibodies and only one of them showed binding of MYBL2 at known target genes (Zhan et al. 2012). Altogether, this highlights the shortcoming of conventional ChIP and why an unbiased method such as TINC, which does not rely on specific antibodies, is of major importance for many scientific fields. Nevertheless, our validation experiments confirmed Nanog promoter binding of these novel factors, some of which, including RCOR2, had previously been identified to play a role in pluripotency. RCOR2 is a subunit of the histone demethylase LSD1 complex in mouse ESCs and it has been shown to be able to replace SOX2 during somatic cell reprogramming into iPSCs (Yang et al. 2011). The authors of this work also showed that knockdown of Rcor2 in ESCs results in significantly decreased expression levels of Nanog and Sox2. Based on RCOR2 ChIP-qPCR for selected differentiation marker genes, the authors proposed an indirect mechanism for the decrease of these pluripotency genes upon RCOR2 depletion. However, our TINC and ChIP data suggest that Nanog is a direct target of RCOR2. The observed decrease in Nanog expression upon RCOR2 depletion (Yang et al. 2011) suggests that RCOR2 acts as transcriptional activator of this pluripotency gene. Similarly, knockout of Zfp57, a transcriptional regulator conventionally associated with maintaining maternal and paternal genomic imprinting, has previously been associated with transcriptional deregulation of 146 genes in mouse ESCs including Nanog (Riso et al. 2016). While the authors did not detect ZFP57 binding within $100 \mathrm{~kb}$ of Nanog and hence did not consider it as one of the genes directly regulated by this TF (Riso et al. 2016) our TINC and ChIP data indicate that Nanog is a direct target of ZFP57. On the other hand, it has previously been shown that knockdown of DNMT3L, a catalytically inactive member of the DNMT3 family of de novo DNA methyltransferases, does not alter the expression of Nanog in mouse ESCs but their differentiation potential (Neri et al. 2013). Together with our data, which revealed binding of this epigenetic modulator at the Nanog promoter in ESCs, this suggests that DNMT3L might play a role in the silencing of Nanog during differentiation; in particular into primordial germinal cells (Neri et al. 2013). Even though the expression of NFATC2IP and WDR7 increases significantly during reprogramming of MEFs into iPSCs (Knaupp et al. 2017), their roles in ESCs remain largely unknown. While NFATC2IP has been associated with transcriptional regulation of cytokine genes in $\mathrm{T}$ helper cells through methylation of histone H4 at arginine 3 (H4R3) in specific cytokine promoters (Fathman et al. 2010), WDR76 might play a role in epigenetic transcriptional regulation as it preferentially binds to 5hydroxymethylcytosine (Spruijt et al. 2013). Our work now suggests that both of these proteins might also play a role in Nanog regulation as we showed (via TINC and ChIP-qPCR) that NFATC2IP and WDR76 associate with its promoter.

Overall, TINC allowed us to identify a large number of proteins associated with functions that are connected to Nanog transcriptional regulation such as stem cell population maintenance, covalent chromatin modifications, nucleosome disassembly and mRNA splicing. There is evidence that pre-mRNA splicing can occur post-transcriptionally but most splicing events seem to take place during the process of transcription (reviewed in (Brugiolo, Herzel, and Neugebauer 2013)). Our data supports this concept, since we detected several proteins associated with mRNA splicing at the Nanog promoter. Besides proteins related to 
transcriptional regulation, we found many proteins associated with nuclear division, DNA replication and double strand break repair some of which might be explained by the fact that the cells were potentially at many different stages of the cell cycle at the point of analysis, as ESCs are self-renewing and constantly proliferating (Lobanok et al. 2009). Additionally, our TINC data provides plausible explanations for several previously published observations. For example, Loh and colleagues showed that depletion of the histone demethylase JMJD1A (also known as KDM3A) in mouse ESCs leads to a significant decrease in Nanog expression (Loh et al. 2007). The authors did not detect JMJD1A at the Nanog promoter in ChIP experiments and consequently attributed this change in Nanog expression to an indirect effect. Our TINC analysis, however, revealed that the Nanog promoter is indeed a direct target of JMJD1A hence suggesting JMJD1A might play a role in histone H3 lysine 9 (H3K9) demethylation in this region.

Furthermore, Gingold and colleagues performed a genome-wide RNAi screen using a Nanog-GFP reporter mouse ESC line under differentiation-promoting conditions to identify factors required for pluripotency maintenance and differentiation (Gingold et al. 2014). Interestingly, we identified 234 of their candidates at the Nanog promoter but only 13 of them were associated with a change in Nanog expression one of which is Zfp57 (Gingold et al. 2014). This suggests that the majority of the proteins that interact with the Nanog promoter are functionally redundant under the conditions tested. As to whether they may play a role during differentiation into cell types not promoted by the author's mild retinoic acid induceddifferentiation conditions, remains elusive. Also, it should be taken into consideration that a change in Nanog expression below the threshold set by the authors (e.g. |Z-score|>2) might still have a significant effect on the cells' self-renewal and/or pluripotency potential. Out of the 13 proteins associated with a change in Nanog expression, knockdown of seven led to an increase (Blm, Ik, Pogz, Pola2, Ppil2, Ppplca and Zfp57) whereas six (Brd4, Cbx1, Chek2, Fen1, Rnh1 and $\mathrm{Wiz}$ ) led to a decrease in Nanog-GFP fluorescence (Gingold et al. 2014). This suggests that transcriptional activators and repressors co-occupy the Nanog promoter. It has previously been shown that some of the Nanog repressors that were found to interact with this RE in ESCs including ZFP281 (Fidalgo et al. 2011) and TCF3 (Yi, Pereira, and Merrill 2008) play a role during differentiation by helping the cell to exit the pluripotent state. Consequently, integration of our TINC data with this RNAi screen potentially suggests that the Nanog promoter is occupied by a large number of proteins whose primary role is not to maintain the pluripotent state but rather to allow exit of this state upon exposure of the cell to specific differentiation cues.

In summary, TINC allowed us to interrogate the regulatory complex formed at the Nanog promoter in an unbiased manner and provided insight into general molecular processes of the cell. In regards to Nanog, our data suggest transcriptional regulation of this key pluripotency TF occurs at many different levels since factors with various functions including DNA modifiers, histone modifiers, chromatin remodelers, transcriptional activators and transcriptional repressors reside at its promoter. Together, this implies a highly complex and coordinated interplay of these factors mediating fine-tuning of the expression of Nanog. Furthermore, our data suggest that many factors that aid in downregulation of Nanog during differentiation already reside at its promoter in the pluripotent state. 


\section{Materials and Methods \\ Cell culture}

ESCs were cultured in gelatin coated dishes at $37^{\circ} \mathrm{C}, 5 \% \quad \mathrm{CO}_{2}$ in ESC medium (KnockOutTM DMEM (Life Technologies) containing 15\% Fetal Bovine Serum (Thermo Scientific), GlutaMAX Supplement (Life Technologies), MEM Non-Essential Amino Acids Solution (Life Technologies), Penicillin-Streptomycin (Life Technologies), 0.1\% (v/v) 2Mercaptoethanol (Life Technologies) and $1000 \mathrm{U} / \mathrm{mL}$ Leukemia Inhibitory Factor (LIF) (Merck Millipore)) supplemented with 2i (1mM PD0325901 (StemMACS) and 3mM CHIR99021 (StemMACS)). To maintain selective pressure, TALE expressing ESCs were cultured in the presence of $3 \mu \mathrm{g} / \mathrm{mL}$ blasticidin (Invitrogen Cat\# R210-01) and ESC lines stably expressing 3xHA-tagged open reading frames were cultured in the presence of $1 \mu \mathrm{g} / \mathrm{mL}$ puromycin (Sigma-Aldrich, Cat\# P8833).

\section{Generation of the TALE-expressing ESC line}

The Nanog targeting TALE was created as described in (Briggs et al. 2012) and inserted into the mammalian expression vector pEF-DEST51 (Life Technologies). The vector was then linearized using SgrDI (Thermo Fisher Scientific) and approximately 1.6 x 10 6 C57BL/6 ESCs were transfected with $15 \mu \mathrm{g}$ of linearized vector using Lipofectamine 3000 (Thermo Fisher Scientific) according to the manufacturer's recommendations. As negative control, the cells were transfected with $15 \mu \mathrm{g}$ of linearized empty pEF-DEST51. $48 \mathrm{~h}$ post transfection, blasticidin (Invitrogen Cat\# R210-01) was added to the cells a final concentration of $3 \mu \mathrm{g} / \mathrm{mL}$ to eliminate any untransfected cells. To create clonal lines, after approximately two weeks colonies were picked manually and cultured under selective pressure. Lines were then analyzed for TALE expression by western blot using an anti HA-tag antibody (Genscript Cat\# A0016840).

\section{Generation of ESC lines stably expressing HA-tagged open reading frames}

Mouse open reading frame (ORF) expression clones in lentiviral vectors with an EF1 $\alpha$ promoter (3xHA-tagged ORF:IRES:mCherry:T2A:puromycin) were obtained for Sox2 (C3xHA-tag; United BioResearch Products), Mybl2 (C-3xHA-tag; VectorBuilder), Rcor2 (C3xHA-tag; VectorBuilder), Wdr76 (N-3xHA tag; VectorBuilder), Dnmt3l (C-3xHA-tag; VectorBuilder), Nfatc2ip (N-3xHA tag; VectorBuilder) and Zfp57 (C-3xHA-tag; VectorBuilder). As controls, we utilized two lentiviral vectors with EF1 $\alpha$ promoter: "mcherry control" (mCherry:T2A:puromycin) and "3xHA-tag control" (3xHAtag:IRES:mCherry:T2A:puromycin) (VectorBuilder). Lentiviruses were created by cotransfecting HEK293T cells with the transgene and packaging vectors (PAX2 and MD2.G) as previously described (Larcombe et al. 2019). Briefly, the media was collected at 48 and $72 \mathrm{~h}$ post transfection, lentiviral particles were concentrated with PEG precipitation and the titers determined by FACS. C57BL/6 ESCs were transduced with the lentivirus at a multiplicity of infection (MOI) ranging from 0.1 to 1 with $5 \mu \mathrm{g} / \mu \mathrm{L}$ polybrene (Merck Millipore, Cat\# TR1003G). From day three onward (e.g. 48 h post transduction), cells were cultured in the presence of $1 \mu \mathrm{g} / \mathrm{mL}$ puromycin (Sigma-Aldrich, Cat\# P8833) to select for transgene integration. Nuclear proteins were isolated using a cell fractionation kit (Cell Signalling 
Technology Cat\# 9038D) and transgene expression was confirmed via western blotting using an anti-HA-tag antibody (Abcam Cat\# ab94918).

\section{Immunofluorescence (IF)}

Cultured cells were fixed in 4\% paraformaldehyde (PFA) (Sigma Aldrich) in PBS for 10 min and then permeabilized in PBS containing 0.3\% Triton X-100 (Sigma Aldrich). Cultures were then incubated with primary antibodies followed by secondary antibodies (see dilutions below). 4',6-diamidino-2-phenylindole (DAPI) (1:1000) (Life Technologies) was added to visualize cell nuclei. Images were taken with an inverted fluorescence microscope and attached camera (Nikon Eclipse Ti). Primary antibodies used in the study were: anti-HA-tag (Cat\# ab94918, Abcam, 1:200), mouse anti-Nanog (Cat\# sc-134218, Santa Cruz, 1:100). Secondary antibodies used in the study (all 1:400) were AF488 donkey anti-goat (Cat\# A32814, Life Technologies), AF555 donkey anti-mouse IgG (Cat\# A31570, Life Technologies).

\section{Teratoma Assays}

Approximately $1.0 \times 10^{6}$ undifferentiated ESCs were injected subcutaneously into the dorsal flanks of immuno-deficient 4 to 20-week-old NOD-SCID mice. Teratomas that developed within 4 weeks post-injection were harvested and fixed in $4 \%$ paraformaldehyde (Merck), embedded in paraffin, sectioned at $5 \mathrm{~mm}$ and stained with haematoxylin and eosin $(\mathrm{H}$ $\&$ E). All animal experimentation was performed under the auspices and approval of the Monash University Animal Research Platform animal ethics committee (Approval Number 14351).

\section{Quantitative RT-PCR}

RNA was extracted using the RNeasy Mini Kit (Qiagen) according to the manufacturer's instructions (with DNase digestion step). RNA was eluted from the columns with $50 \mu \mathrm{L}$ RNase-free water and quantified using a Nanodrop (Nanodrop Technologies). 500 ng of RNA were then used for cDNA synthesis using the SuperScript III First-Strand Synthesis kit (Invitrogen) according to the manufacturer's instructions. Real-time quantitative PCR reactions were set up in triplicate with $2 \mu \mathrm{L}$ of cDNA (1:4 dilution) per reaction using the FastStart Universal SYBR Green Master mix (Rox) (Roche) and run on a 7500 Real-Time PCR System (Applied Biosystems). Gene expression levels were calculated using the $2^{-\Delta \Delta \mathrm{CT}}$ method with Gapdh as internal control. The following primer sequences (5'-3') were used: Gapdh (F: AGGTCGGTGTGAACGGATTTG, R: TGTAGACCATGTAGTTGAGGTCA) and Nanog (F: TTGCTTACAAGGGTCTGCTACT, R: ACTGGTAGAAGAATCAGGGCT).

\section{Chromatin Immunoprecipitation qPCR}

ChIPs were conducted as described by us previously (Knaupp et al. 2017) with slight modifications. Approximately $30 \times 10^{6}$ cells were used per ChIP. The samples were pre-cleared with $100 \mu \mathrm{L}$ of Pierce Control Agarose Resin (Thermo Fisher Scientific) for $1 \mathrm{~h}$ at $4{ }^{\circ} \mathrm{C}$ and immunoprecipitations were performed with $30 \mu \mathrm{L}$ of Pierce Anti-HA Agarose (Thermo Fisher Scientific) per sample rotating overnight at $4{ }^{\circ} \mathrm{C}$. After DNA purification using the QIAquick PCR Purification Kit (Qiagen Cat\# 28106), real-time quantitative PCR reactions were set up 
in triplicate. $2 \mu \mathrm{L}$ of DNA were used per reaction with the FastStart Universal SYBR Green Master mix (Rox) (Roche) and run on a 7500 Real-Time PCR System (Applied Biosystems). The percent of input was calculated for each ChIP $\left(\%\right.$ Input $\left.=2^{(-\Delta \mathrm{Ct} \text { [normalized ChIP] })}\right)$. The following primer sequences (5'-3') were used: Nanog promoter (F: AATGAGGTAAAGCCTCTTTTTGG, R: ACCATGGACATTGTAATGCAAA) and background region (e.g. Sox2 regulatory region 2) (F: GCTAGGCAGGTTCCCCTCTA, R: GCAAGAACTGTCGACTGTGC).

\section{TALE-mediated Isolation of Nuclear Chromatin (TINC)}

Approximately $1 \times 10^{9}$ cells were fixed with formaldehyde as described by us previously (Knaupp et al. 2017) and nuclei and chromatin were isolated according to Kustatscher et al., 2014 to remove non-chromatin-associated proteins. The chromatin was then resuspended in ChIP lysis buffer (1\% SDS, $10 \mathrm{mM}$ EDTA, $50 \mathrm{mM}$ Tris $\mathrm{pH}$ 8.0) containing protease inhibitor cocktail (Sigma-Aldrich Cat\# P3840) at $1 \mathrm{~g}$ of chromatin per $3.2 \mathrm{~mL}$ of buffer and sonicated on a Bioruptor NextGen sonication device (Diagenode) to shear the chromatin to an average fragment size of $200-500 \mathrm{bp}$ as determined by agarose gel electrophoresis. The samples were then diluted 1:5 with ChIP dilution buffer (165 mM NaCl, 0.01\% SDS, $1.1 \%$ Triton X-100, 1.2 mM EDTA, $16.7 \mathrm{mM}$ Tris $\mathrm{pH}$ 8.0) containing protease inhibitor cocktail (Sigma-Aldrich Cat\# P3840) and 0.05\% input was collected and put aside on ice. Additionally, $0.05 \%$ of the sample was used to determine the DNA concentration to ensure the same amount of chromatin was used for the TALE and the control samples in the TINC assay. To do so, the cross-linking was reversed for $15 \mathrm{~min}$ at $100{ }^{\circ} \mathrm{C}$, the sample purified using a QIAquick PCR Purification Kit (Qiagen) and DNA concentration determined using the Qubit dsDNA HS Assay Kit (Thermo Fisher Scientific) and a Qubit 2.0 Fluorometer (Thermo Fisher Scientific). The samples were then precleared with $1 \mathrm{~mL}$ of settled Pierce Control Agarose Resin (Thermo Fisher Scientific Cat\#26150) for $1 \mathrm{~h}$ at $4{ }^{\circ} \mathrm{C}$.

TALE-immunoprecipitations were performed with $1 \mathrm{~mL}$ of settled Pierce Anti-HA Agarose Resin (Thermo Fisher Scientific Cat\# 26182) overnight at $4{ }^{\circ} \mathrm{C}$. Samples were then washed twice for $10 \mathrm{~min}$ at $4{ }^{\circ} \mathrm{C}$ with dilution buffer containing protease inhibitor cocktail, followed by two washes for $10 \mathrm{~min}$ at $4{ }^{\circ} \mathrm{C}$ with low salt buffer $(150 \mathrm{mM} \mathrm{NaCl}, 0.5 \% \mathrm{Na}$ deoxycholate, $0.1 \%$ SDS, $1 \%$ Nonidet P-40, $1 \mathrm{mM}$ EDTA, $50 \mathrm{mM}$ Tris pH8) containing protease inhibitor cocktail, two washes for $10 \mathrm{~min}$ at $4{ }^{\circ} \mathrm{C}$ with high salt buffer $(500 \mathrm{mM} \mathrm{NaCl}$, $0.5 \% \mathrm{Na}$ deoxycholate, $0.1 \%$ SDS, $1 \%$ Nonidet P-40, $1 \mathrm{mM}$ EDTA, 50mM Tris $\mathrm{pH} 8$ ) and a final $5 \mathrm{~min}$ wash at $4{ }^{\circ} \mathrm{C}$ with TE buffer $(0.25 \mathrm{mM}$ EDTA, $10 \mathrm{mM}$ Tris $\mathrm{pH}$ 8). The volume was $100 \mathrm{~mL}$ for each wash step. For elution, the samples were resuspended in $2 \mathrm{~mL}$ of $3 \mathrm{M} \mathrm{NaSCN}$ and incubated rotating for $5 \mathrm{~min}$ at room temperature. The eluted samples were removed and the elution step was repeated three times. All four elutions were pooled and concentrated using $3 \mathrm{KDa}$ cut-off concentrators (Amicon Ultra Cat\# UFC800308 and UFC500308) to $50 \mu \mathrm{L}$. During concentration, the samples were diluted 1:20 with PBS to dilute the NaSCN concentration. $1 \mu \mathrm{L}$ per sample was then removed and the DNA of this sample as well as of the input samples was then purified as described above to ensure enrichment of the Nanog promoter as tested by qPCR as described in the ChIP-qPCR section. The remaining sample was subjected to LC-MS/MS analysis. 


\section{LC-MS/MS analysis}

To determine the proteins isolated by TINC, 4 x loading dye (40\% glycerol, $4 \%$ SDS, $400 \mathrm{mM}$ DTT, $0.04 \%$ bromophenol blue, $125 \mathrm{mM}$ Tris $\mathrm{pH} 8.3$ ) was added to the samples and the samples were incubated at $100^{\circ} \mathrm{C}$ for $30 \mathrm{~min}$. The samples were then loaded onto a NuPAGE 4-12\% Bis-Tris Protein Gel (Thermo Fisher Scientific Cat\# NP0335BOX) and a short gel was run using NuPAGE MOPS SDS Running Buffer (Thermo Fisher Scientific Cat\# NP0001) to stack the proteins. The proteins were visualized with InstantBlue Coomassie Protein Stain (Expedeon Cat\#ISB1L) and the according band excised from the gel. The proteins were reduced with $5 \mathrm{mM}$ DTT (Agilent Technologies Cat\# 5188-6439), alkylated with $18 \mathrm{mM}$ 2-chloroacetamide (Sigma-Aldrich Cat\# C0267-100G) and in-gel digested overnight at $37^{\circ} \mathrm{C}$ with sequencing grade trypsin (Promega Cat\# V5111). The peptides were then extracted with extraction buffer (50\% acetonitrile (Fisher Chemical Cat\#A955-4), 5\% formic acid (SigmaAldrich Cat\# 56302-50ML-F)) followed by extraction with $100 \%$ acetonitrile. All extracted peptides were combined and dried to completion in an CentriVap Concentrator (Labconco). Samples were then resuspended in $2 \%$ acetonitrile, $0.1 \%$ formic acid prior to LC-MS/MS analysis.

Using a Dionex UltiMate 3000 RSLCnano system equipped with a Dionex UltiMate 3000 RS autosampler, the samples were loaded via an Acclaim PepMap 100 trap column (100 $\mu \mathrm{m}$ x $2 \mathrm{~cm}$, nanoViper, C18, $5 \mu \mathrm{m}, 100 a ̊$; Thermo Scientific) onto an Acclaim PepMap RSLC analytical column (75 $\mu \mathrm{m}$ x $50 \mathrm{~cm}$, nanoViper, C18, $2 \mu \mathrm{m}, 100 \mathrm{a}$; Thermo Scientific). The tryptic peptides were separated by increasing concentrations of $80 \%$ acetonitrile (CAN) / $0.1 \%$ FA at a flow of $250 \mathrm{nl} / \mathrm{min}$ for $158 \mathrm{~min}$ and analyzed with an Orbitrap Fusion Tribrid mass spectrometer (Thermo Scientific). Each cycle was set to a fixed cycle time of 2 sec consisting of an Orbitrap full ms1 scan (resolution: 120.000; AGC target: 1e6; maximum IT: 54 ms; scan range: $375-1575 \mathrm{~m} / \mathrm{z}$ ) followed by several Orbitrap ms2 scans (resolution: 30.000; AGC target: 2e5; maximum IT: $54 \mathrm{~ms}$; isolation window: $1.4 \mathrm{~m} / \mathrm{z}$; HCD Collision Energy: 32\%). To minimize repeated sequencing of the same peptides, the dynamic exclusion was set to $15 \mathrm{sec}$ and the 'exclude isotopes' option was activated.

Acquired .raw files were searched against the murine UniProtKB/SwissProt database (v2017_07) appended with the TALE protein sequence using Byonic (Protein Metrics) considering a false discovery rate (FDR) of $1 \%$ using the target-decoy approach. Carbamidomethylation of cysteine residues was specified as a fixed modification. Oxidation of methionine and acetylation of protein N-termini were selected as variable modifications. Trypsin was used as the enzymatic protease and up to two missed cleavages were allowed. Data visualization and mining was performed in Excel. To minimize false positive protein identifications, we only considered proteins that were identified by at least two unique peptides.

\section{Data mining}

For validation of Nanog promoter interactors identified by TINC, ChIP-ATLAS was used for data mining of published ChIP-seq datasets (Oki et al. 2018). Datasets were chosen on the basis of binding between the region $1 \mathrm{~kb}$ upstream of the Nanog enhancer, and the 3' UTR region of Nanog. Furthermore the datasets had to be of untreated/control mouse ESCs. BigWig files and peak files were obtained pre-processed by the ChIP-ATLAS pipeline. The narrow peak files obtained, containing peaks with a false discovery rate cut-off of 0.05 . These 
files were converted into BigBed files using Homer (Heinz et al. 2010). All BigBed and BigWig files were visualised with UCSC mm9 (W. James Kent et al. 2002; W. J. Kent et al. 2010; Raney et al. 2014). For the validation, the data with the following accession numbers was used: SRX2245718, SRX1158299, SRX997153, ERX2244637, SRX039347, SRX4167129，SRX1738884，SRX236477，SRX2441332，SRX1590979，SRX017058, SRX1342343， SRX003864，SRX319555，SRX4394631，SRX476210， SRX2831314, SRX2831316, and SRX017060.

\section{Enrichment and other statistical analyses}

Protein classification analyses were performed with Panther (Thomas et al. 2003), and all other ontology analyses were performed with Metascape (Zhou et al. 2019). Transcription factors were defined by collected annotation from Riken and fantom five mouse TF datasets (Carninci et al. 2005; Kanamori et al. 2004). All other epigenetic modifiers and respective metadata were obtained from the epifactors database (Medvedeva et al. 2015). The pluripotency network was obtained from ESCAPE (Embryonic Stem Cells Atlas of Pluripotency Evidence) (Xu et al. 2013, 2014). Enrichment of TINC proteins within the pluripotency network was calculated with Fisher's exact test. RNA-seq data of reprogramming fibroblasts into iPSCs was obtained from Knaupp et al. 2017. Raw sequencing files were processed to raw counts as described in the original publication. The raw counts were then TMM normalized and converted to $\log 2$ CPM for all analyses. The standardized log2CPM values were centred to the mean expression for each gene. Pearson's correlation coefficients were calculated on standardized $\log 2 \mathrm{CPM}$ values for all genes corresponding to proteins identified in all three TINC replicates. Protein-protein enrichment networks were visualized with Cytoscape v3.7.1 (Shannon et al. 2003). ComplexHeatmap was used for any visualization of heatmaps with default clustering parameters and all other analyses outputs were graphed using GGplot2 unless otherwise stated (Gu, Eils, and Schlesner 2016; Wickham 2016). A set seed of 123 was used in all analyses, unless otherwise stated. Protein-protein interaction network functional enrichment analysis (high confidence (0.700) interaction score) and kmeans clustering was conducted using STRING version 11.0 (Szklarczyk et al. 2019). Clusters identified were subjected to gene ontology enrichment using Metascape (Zhou et al. 2019) to identify the most significantly enriched terms for each cluster. Venn diagrams were produced using BioVenn (Hulsen, de Vlieg, and Alkema 2008) or jvenn (Bardou et al. 2014).

\section{Acknowledgements}

This work was supported by the National Health and Medical Research Council (NHMRC) of Australia (GNT1069830) and the Australian Research Council (ARC) Centre of Excellence program in Plant Energy Biology (CE140100008). J.M.P. and R.L. are part of the ARC funded Stem Cells Australia Special Initiative and were supported by Sylvia and Charles Viertel Senior Medical Research Fellowships (J.M.P. and R.L.), NHMRC CDF (J.M.P., APP1036587), ARC Future Fellowship (R.L., FT120100862), and Howard Hughes Medical Institute International Research Scholarship (R.L.). A.S.K. was supported by an NHMRC Early Career Fellowship (APP1092280). We would like to acknowledge the Monash University Histology Platform Paraffin Laboratory for the provision of reagents and technical support. 


\section{References}

Abranches, Elsa, Ana M. V. Guedes, Martin Moravec, Hedia Maamar, Petr Svoboda, Arjun Raj, and Domingos Henrique. 2014. "Stochastic NANOG Fluctuations Allow Mouse Embryonic Stem Cells to Explore Pluripotency." Development 141 (14): 2770-79.

Antão, José M., James M. Mason, Jérôme Déjardin, and Robert E. Kingston. 2012. "Protein Landscape at Drosophila Melanogaster Telomere-Associated Sequence Repeats." Molecular and Cellular Biology 32 (12): 2170-82.

Apostolou, Effie, Francesco Ferrari, Ryan M. Walsh, Ori Bar-Nur, Matthias Stadtfeld, Sihem Cheloufi, Hannah T. Stuart, et al. 2013. "Genome-Wide Chromatin Interactions of the Nanog Locus in Pluripotency, Differentiation, and Reprogramming." Cell Stem Cell 12 (6): 699-712.

Bardou, Philippe, Jérôme Mariette, Frédéric Escudié, Christophe Djemiel, and Christophe Klopp. 2014. "Jvenn: An Interactive Venn Diagram Viewer." BMC Bioinformatics 15 (August): 293.

Boyer, Laurie A., Tong Ihn Lee, Megan F. Cole, Sarah E. Johnstone, Stuart S. Levine, Jacob P. Zucker, Matthew G. Guenther, et al. 2005. "Core Transcriptional Regulatory Circuitry in Human Embryonic Stem Cells.” Cell 122 (6): 947-56.

Briggs, Adrian W., Xavier Rios, Raj Chari, Luhan Yang, Feng Zhang, Prashant Mali, and George M. Church. 2012. "Iterative Capped Assembly: Rapid and Scalable Synthesis of Repeat-Module DNA such as TAL Effectors from Individual Monomers." Nucleic Acids Research 40 (15): e117.

Brugiolo, Mattia, Lydia Herzel, and Karla M. Neugebauer. 2013. "Counting on CoTranscriptional Splicing." F1000prime Reports 5 (April): 9.

Byrum, Stephanie D., Ana Raman, Sean D. Taverna, and Alan J. Tackett. 2012. "ChAP-MS: A Method for Identification of Proteins and Histone Posttranslational Modifications at a Single Genomic Locus." Cell Reports. https://doi.org/10.1016/j.celrep.2012.06.019.

Byrum, Stephanie D., Sean D. Taverna, and Alan J. Tackett. 2013. "Purification of a Specific Native Genomic Locus for Proteomic Analysis." Nucleic Acids Research 41 (20): e195.

Carninci, P., T. Kasukawa, S. Katayama, J. Gough, M. C. Frith, N. Maeda, R. Oyama, et al. 2005. "The Transcriptional Landscape of the Mammalian Genome." Science 309 (5740): 1559-63.

Carter, Ava C., Brandi N. Davis-Dusenbery, Kathryn Koszka, Justin K. Ichida, and Kevin Eggan. 2014. "Nanog-Independent Reprogramming to iPSCs with Canonical Factors." Stem Cell Reports 2 (2): 119-26.

Chambers, Ian, Douglas Colby, Morag Robertson, Jennifer Nichols, Sonia Lee, Susan Tweedie, and Austin Smith. 2003. "Functional Expression Cloning of Nanog, a Pluripotency Sustaining Factor in Embryonic Stem Cells." Cell 113 (5): 643-55.

Chambers, Ian, Jose Silva, Douglas Colby, Jennifer Nichols, Bianca Nijmeijer, Morag Robertson, Jan Vrana, Ken Jones, Lars Grotewold, and Austin Smith. 2007. "Nanog Safeguards Pluripotency and Mediates Germline Development." Nature 450 (7173): 1230-34.

Chen, Xi, Han Xu, Ping Yuan, Fang Fang, Mikael Huss, Vinsensius B. Vega, Eleanor Wong, et al. 2008. "Integration of External Signaling Pathways with the Core Transcriptional Network in Embryonic Stem Cells." Cell 133 (6): 1106-17.

Collas, Philippe. 2010. "The Current State of Chromatin Immunoprecipitation." Molecular Biotechnology 45 (1): 87-100.

Déjardin, Jérôme, and Robert E. Kingston. 2009. "Purification of Proteins Associated with Specific Genomic Loci.” Cell. https://doi.org/10.1016/j.cell.2008.11.045.

Fang, Fang, Ninuo Xia, Benjamin Angulo, Joseph Carey, Zackery Cady, Jens Durruthy- 
Durruthy, Theo Bennett, Vittorio Sebastiano, and Renee A. Reijo Pera. 2018. "A Distinct Isoform of ZNF207 Controls Self-Renewal and Pluripotency of Human Embryonic Stem Cells." Nature Communications 9 (1): 4384.

Fathman, John W., Michael F. Gurish, Saskia Hemmers, Kevin Bonham, Daniel S. Friend, Michael J. Grusby, Laurie H. Glimcher, and Kerri A. Mowen. 2010. "NIP45 Controls the Magnitude of the Type 2 T Helper Cell Response." Proceedings of the National Academy of Sciences of the United States of America 107 (8): 3663-68.

Fidalgo, Miguel, Francesco Faiola, Carlos-Filipe Pereira, Junjun Ding, Arven Saunders, Julian Gingold, Christoph Schaniel, Ihor R. Lemischka, José C. R. Silva, and Jianlong Wang. 2012. "Zfp281 Mediates Nanog Autorepression through Recruitment of the NuRD Complex and Inhibits Somatic Cell Reprogramming." Proceedings of the National Academy of Sciences of the United States of America 109 (40): 16202-7.

Fidalgo, Miguel, P. Chandra Shekar, Yen-Sin Ang, Yuko Fujiwara, Stuart H. Orkin, and Jianlong Wang. 2011. "Zfp281 Functions as a Transcriptional Repressor for Pluripotency of Mouse Embryonic Stem Cells.” Stem Cells 29 (11): 1705-16.

Fujita, Toshitsugu, Yoshinori Asano, Junko Ohtsuka, Yoko Takada, Kazunobu Saito, Rieko Ohki, and Hodaka Fujii. 2013. "Identification of Telomere-Associated Molecules by Engineered DNA-Binding Molecule-Mediated Chromatin Immunoprecipitation (enChIP)." Scientific Reports 3 (November): 3171.

Fujita, Toshitsugu, and Hodaka Fujii. 2011. "Direct Identification of Insulator Components by Insertional Chromatin Immunoprecipitation.” PloS One 6 (10): e26109.

. 2013. "Efficient Isolation of Specific Genomic Regions and Identification of Associated Proteins by Engineered DNA-Binding Molecule-Mediated Chromatin Immunoprecipitation (enChIP) Using CRISPR." Biochemical and Biophysical Research Communications 439 (1): 132-36.

Furey, Terrence S. 2012. "ChIP-Seq and beyond: New and Improved Methodologies to Detect and Characterize Protein-DNA Interactions." Nature Reviews. Genetics 13 (12): 840-52.

Gao, Xin D., Li-Chun Tu, Aamir Mir, Tomás Rodriguez, Yuehe Ding, John Leszyk, Job Dekker, et al. 2018. "C-BERST: Defining Subnuclear Proteomic Landscapes at Genomic Elements with dCas9-APEX2." Nature Methods. https://doi.org/10.1038/s41592-0180006-2.

Gingold, Julian A., Miguel Fidalgo, Diana Guallar, Zerlina Lau, Zhen Sun, Hongwei Zhou, Francesco Faiola, et al. 2014. "A Genome-Wide RNAi Screen Identifies Opposing Functions of Snai1 and Snai2 on the Nanog Dependency in Reprogramming." Molecular Cell 56 (1): 140-52.

Gu, Zuguang, Roland Eils, and Matthias Schlesner. 2016. "Complex Heatmaps Reveal Patterns and Correlations in Multidimensional Genomic Data." Bioinformatics 32 (18): 2847-49.

Heinz, Sven, Christopher Benner, Nathanael Spann, Eric Bertolino, Yin C. Lin, Peter Laslo, Jason X. Cheng, Cornelis Murre, Harinder Singh, and Christopher K. Glass. 2010. "Simple Combinations of Lineage-Determining Transcription Factors Prime CisRegulatory Elements Required for Macrophage and B Cell Identities." Molecular Cell 38 (4): 576-89.

Hulsen, Tim, Jacob de Vlieg, and Wynand Alkema. 2008. "BioVenn - a Web Application for the Comparison and Visualization of Biological Lists Using Area-Proportional Venn Diagrams." BMC Genomics. https://doi.org/10.1186/1471-2164-9-488.

Ide, Satoru, and Jerome Dejardin. 2015. "End-Targeting Proteomics of Isolated Chromatin Segments of a Mammalian Ribosomal RNA Gene Promoter." Nature Communications 6 (March): 6674.

Kagey, Michael H., Jamie J. Newman, Steve Bilodeau, Ye Zhan, David A. Orlando, Nynke L. van Berkum, Christopher C. Ebmeier, et al. 2010. "Mediator and Cohesin Connect Gene 
Expression and Chromatin Architecture." Nature 467 (7314): 430-35.

Kalmar, Tibor, Chea Lim, Penelope Hayward, Silvia Muñoz-Descalzo, Jennifer Nichols, Jordi Garcia-Ojalvo, and Alfonso Martinez Arias. 2009. "Regulated Fluctuations in Nanog Expression Mediate Cell Fate Decisions in Embryonic Stem Cells.” PLoS Biology 7 (7): e1000149.

Kanamori, Mutsumi, Hideaki Konno, Naoki Osato, Jun Kawai, Yoshihide Hayashizaki, and Harukazu Suzuki. 2004. "A Genome-Wide and Nonredundant Mouse Transcription Factor Database." Biochemical and Biophysical Research Communications 322 (3): 78793.

Kaufman, M. H., E. J. Robertson, A. H. Handyside, and M. J. Evans. 1983. "Establishment of Pluripotential Cell Lines from Haploid Mouse Embryos." Journal of Embryology and Experimental Morphology 73 (February): 249-61.

Kennedy-Darling, Julia, Hector Guillen-Ahlers, Michael R. Shortreed, Mark Scalf, Brian L. Frey, Christina Kendziorski, Michael Olivier, Audrey P. Gasch, and Lloyd M. Smith. 2014. "Discovery of Chromatin-Associated Proteins via Sequence-Specific Capture and Mass Spectrometric Protein Identification in Saccharomyces Cerevisiae." Journal of Proteome Research 13 (8): 3810-25.

Kent, W. James, Charles W. Sugnet, Terrence S. Furey, Krishna M. Roskin, Tom H. Pringle, Alan M. Zahler, and David Haussler. 2002. "The Human Genome Browser at UCSC." Genome Research 12 (6): 996-1006.

Kent, W. J., A. S. Zweig, G. Barber, A. S. Hinrichs, and D. Karolchik. 2010. "BigWig and BigBed: Enabling Browsing of Large Distributed Datasets." Bioinformatics 26 (17): 2204-7.

Kim, Jonghwan, Jianlin Chu, Xiaohua Shen, Jianlong Wang, and Stuart H. Orkin. 2008. "An Extended Transcriptional Network for Pluripotency of Embryonic Stem Cells." Cell 132 (6): 1049-61.

Knaupp, Anja S., Sam Buckberry, Jahnvi Pflueger, Sue Mei Lim, Ethan Ford, Michael R. Larcombe, Fernando J. Rossello, et al. 2017. "Transient and Permanent Reconfiguration of Chromatin and Transcription Factor Occupancy Drive Reprogramming." Cell Stem Cell 21 (6): 834-45.e6.

Kumar, Roshan M., Patrick Cahan, Alex K. Shalek, Rahul Satija, Ajay DaleyKeyser, Hu Li, Jin Zhang, et al. 2014. "Deconstructing Transcriptional Heterogeneity in Pluripotent Stem Cells." Nature 516 (7529): 56-61.

Kustatscher, Georg, Karen L. H. Wills, Cristina Furlan, and Juri Rappsilber. 2014. "Chromatin Enrichment for Proteomics." Nature Protocols 9 (9): 2090-99.

Larcombe, Michael R., Jan Manent, Joseph Chen, Ketan Mishra, Xiaodong Liu, and Christian M. Nefzger. 2019. "Production of High-Titer Lentiviral Particles for Stable Genetic Modification of Mammalian Cells." Methods in Molecular Biology 1940: 47-61.

Liu, W., P. Stein, X. Cheng, W. Yang, N-Y Shao, E. E. Morrisey, R. M. Schultz, and J. You. 2014. "BRD4 Regulates Nanog Expression in Mouse Embryonic Stem Cells and Preimplantation Embryos." Cell Death and Differentiation 21 (12): 1950-60.

Liu, Xin, Yuannyu Zhang, Yong Chen, Mushan Li, Feng Zhou, Kailong Li, Hui Cao, et al. 2017. "In Situ Capture of Chromatin Interactions by Biotinylated dCas9." Cell 170 (5): 1028-43.e19.

Liu, Yinyin, Hyeung Kim, Jiancong Liang, Weisi Lu, Bin Ouyang, Dan Liu, and Zhou Songyang. 2014. "The Death-Inducer Obliterator 1 (Dido1) Gene Regulates Embryonic Stem Cell Self-Renewal." The Journal of Biological Chemistry 289 (8): 4778-86.

Lobanok, E. S., L. M. Mezhevikina, L. M. Belyanovich, R. R. Petrova, I. B. Vasilevich, I. D. Volotovsky, and E. E. Fesenko. 2009. "The Influence of LIF (Leukemia Inhibitory Factor) on the Functional Status of R1 Mouse Embryonic Stem Cells." Biochemistry (Moscow) 
Supplement Series Biomedical Chemistry. https://doi.org/10.1134/s1990750809010077.

Loh, Yuin-Han, Qiang Wu, Joon-Lin Chew, Vinsensius B. Vega, Weiwei Zhang, Xi Chen, Guillaume Bourque, et al. 2006. "The Oct4 and Nanog Transcription Network Regulates Pluripotency in Mouse Embryonic Stem Cells.” Nature Genetics 38 (4): 431-40.

Loh, Yuin-Han, Weiwei Zhang, Xi Chen, Joshy George, and Huck-Hui Ng. 2007. "Jmjd1a and Jmjd2c Histone H3 Lys 9 Demethylases Regulate Self-Renewal in Embryonic Stem Cells." Genes \& Development 21 (20): 2545-57.

Marson, Alexander, Stuart S. Levine, Megan F. Cole, Garrett M. Frampton, Tobias Brambrink, Sarah Johnstone, Matthew G. Guenther, et al. 2008. "Connecting microRNA Genes to the Core Transcriptional Regulatory Circuitry of Embryonic Stem Cells." Cell 134 (3): 52133.

Martin, G. R. 1981. "Isolation of a Pluripotent Cell Line from Early Mouse Embryos Cultured in Medium Conditioned by Teratocarcinoma Stem Cells." Proceedings of the National Academy of Sciences of the United States of America 78 (12): 7634-38.

Medvedeva, Yulia A., Andreas Lennartsson, Rezvan Ehsani, Ivan V. Kulakovskiy, Ilya E. Vorontsov, Pouda Panahandeh, Grigory Khimulya, Takeya Kasukawa, FANTOM Consortium, and Finn Drabløs. 2015. "EpiFactors: A Comprehensive Database of Human Epigenetic Factors and Complexes." Database: The Journal of Biological Databases and Curation 2015 (July): bav067.

Mitsui, Kaoru, Yoshimi Tokuzawa, Hiroaki Itoh, Kohichi Segawa, Mirei Murakami, Kazutoshi Takahashi, Masayoshi Maruyama, Mitsuyo Maeda, and Shinya Yamanaka. 2003. "The Homeoprotein Nanog Is Required for Maintenance of Pluripotency in Mouse Epiblast and ES Cells." Cell 113 (5): 631-42.

Navarro, Pablo, Nicola Festuccia, Douglas Colby, Alessia Gagliardi, Nicholas P. Mullin, Wensheng Zhang, Violetta Karwacki-Neisius, et al. 2012. "OCT4/SOX2-Independent Nanog Autorepression Modulates Heterogeneous Nanog Gene Expression in Mouse ES Cells." The EMBO Journal 31 (24): 4547-62.

Nefzger, Christian M., Fernando J. Rossello, Joseph Chen, Xiaodong Liu, Anja S. Knaupp, Jaber Firas, Jacob M. Paynter, et al. 2017. "Cell Type of Origin Dictates the Route to Pluripotency." Cell Reports 21 (10): 2649-60.

Neri, Francesco, Anna Krepelova, Danny Incarnato, Mara Maldotti, Caterina Parlato, Federico Galvagni, Filomena Matarese, Hendrik G. Stunnenberg, and Salvatore Oliviero. 2013. "Dnmt3L Antagonizes DNA Methylation at Bivalent Promoters and Favors DNA Methylation at Gene Bodies in ESCs." Cell 155 (1): 121-34.

Oki, Shinya, Tazro Ohta, Go Shioi, Hideki Hatanaka, Osamu Ogasawara, Yoshihiro Okuda, Hideya Kawaji, Ryo Nakaki, Jun Sese, and Chikara Meno. 2018. "ChIP-Atlas: A DataMining Suite Powered by Full Integration of Public ChIP-Seq Data." EMBO Reports 19 (12). https://www.embopress.org/doi/abs/10.15252/embr.201846255.

Pourfarzad, Farzin, Ali Aghajanirefah, Ernie de Boer, Sara Ten Have, Thamar Bryn van Dijk, Sima Kheradmandkia, Ralph Stadhouders, et al. 2013. "Locus-Specific Proteomics by TChP: Targeted Chromatin Purification." Cell Reports. https://doi.org/10.1016/j.celrep.2013.07.004.

Qiu, Wenqing, Zhijiao Xu, Min Zhang, Dandan Zhang, Hui Fan, Taotao Li, Qianfeng Wang, et al. 2019. "Determination of Local Chromatin Interactions Using a Combined CRISPR and Peroxidase APEX2 System." Nucleic Acids Research 47 (9): e52.

Raney, Brian J., Timothy R. Dreszer, Galt P. Barber, Hiram Clawson, Pauline A. Fujita, Ting Wang, Ngan Nguyen, et al. 2014. "Track Data Hubs Enable Visualization of User-Defined Genome-Wide Annotations on the UCSC Genome Browser." Bioinformatics 30 (7): $1003-5$. 
Riso, Vincenzo, Marco Cammisa, Harpreet Kukreja, Zahra Anvar, Gaetano Verde, Angela Sparago, Basilia Acurzio, et al. 2016. "ZFP57 Maintains the Parent-of-Origin-Specific Expression of the Imprinted Genes and Differentially Affects Non-Imprinted Targets in Mouse Embryonic Stem Cells." Nucleic Acids Research 44 (17): 8165-78.

Rodda, David J., Joon-Lin Chew, Leng-Hiong Lim, Yuin-Han Loh, Bei Wang, Huck-Hui Ng, and Paul Robson. 2005. "Transcriptional Regulation of Nanog by OCT4 and SOX2." The Journal of Biological Chemistry 280 (26): 24731-37.

Sardina, Jose Luis, Samuel Collombet, Tian V. Tian, Antonio Gómez, Bruno Di Stefano, Clara Berenguer, Justin Brumbaugh, et al. 2018. "Transcription Factors Drive Tet2-Mediated Enhancer Demethylation to Reprogram Cell Fate." Cell Stem Cell 23 (6): 905-6.

Schmidtmann, Elisabeth, Tobias Anton, Pascaline Rombaut, Franz Herzog, and Heinrich Leonhardt. 2016. "Determination of Local Chromatin Composition by CasID." Nucleus 7 (5): 476-84.

Shannon, Paul, Andrew Markiel, Owen Ozier, Nitin S. Baliga, Jonathan T. Wang, Daniel Ramage, Nada Amin, Benno Schwikowski, and Trey Ideker. 2003. "Cytoscape: A Software Environment for Integrated Models of Biomolecular Interaction Networks." Genome Research 13 (11): 2498-2504.

Silva, José, Ian Chambers, Steven Pollard, and Austin Smith. 2006. "Nanog Promotes Transfer of Pluripotency after Cell Fusion.” Nature 441 (7096): 997-1001.

Silva, Jose, Jennifer Nichols, Thorold W. Theunissen, Ge Guo, Anouk L. van Oosten, Ornella Barrandon, Jason Wray, Shinya Yamanaka, Ian Chambers, and Austin Smith. 2009. "Nanog Is the Gateway to the Pluripotent Ground State." Cell 138 (4): 722-37.

Spruijt, Cornelia G., Felix Gnerlich, Arne H. Smits, Toni Pfaffeneder, Pascal W. T. C. Jansen, Christina Bauer, Martin Münzel, et al. 2013. "Dynamic Readers for 5(hydroxy)methylcytosine and Its Oxidized Derivatives." Cell 152 (5): 1146-59.

Strogantsev, Ruslan, Felix Krueger, Kazuki Yamazawa, Hui Shi, Poppy Gould, Megan Goldman-Roberts, Kirsten McEwen, Bowen Sun, Roger Pedersen, and Anne C. FergusonSmith. 2015. "Allele-Specific Binding of ZFP57 in the Epigenetic Regulation of Imprinted and Non-Imprinted Monoallelic Expression." Genome Biology 16 (May): 112.

Szklarczyk, Damian, Annika L. Gable, David Lyon, Alexander Junge, Stefan Wyder, Jaime Huerta-Cepas, Milan Simonovic, et al. 2019. "STRING v11: Protein-protein Association Networks with Increased Coverage, Supporting Functional Discovery in Genome-Wide Experimental Datasets." Nucleic Acids Research 47 (D1): D607-13.

Takahashi, Kazutoshi, and Shinya Yamanaka. 2006. "Induction of Pluripotent Stem Cells from Mouse Embryonic and Adult Fibroblast Cultures by Defined Factors.” Cell 126 (4): 66376.

Thomas, Paul D., Michael J. Campbell, Anish Kejariwal, Huaiyu Mi, Brian Karlak, Robin Daverman, Karen Diemer, Anushya Muruganujan, and Apurva Narechania. 2003. "PANTHER: A Library of Protein Families and Subfamilies Indexed by Function." Genome Research 13 (9): 2129-41.

Tsui, Chiahao, Carla Inouye, Michaella Levy, Andrew Lu, Laurence Florens, Michael P. Washburn, and Robert Tjian. 2018. "dCas9-Targeted Locus-Specific Protein Isolation Method Identifies Histone Gene Regulators." Proceedings of the National Academy of Sciences of the United States of America 115 (12): E2734-41.

Vermeulen, Michiel, and Jérôme Déjardin. 2020. "Publisher Correction: Locus-Specific Chromatin Isolation." Nature Reviews. Molecular Cell Biology, February. https://doi.org/10.1038/s41580-020-0222-3.

Waldrip, Zachary J., Stephanie D. Byrum, Aaron J. Storey, Jun Gao, Alicia K. Byrd, Samuel G. Mackintosh, Wayne P. Wahls, Sean D. Taverna, Kevin D. Raney, and Alan J. Tackett. 2014. "A CRISPR-Based Approach for Proteomic Analysis of a Single Genomic Locus." 
Epigenetics: Official Journal of the DNA Methylation Society 9 (9): 1207-11.

Wang, Zheng-Xu, Christina Hui-Leng Teh, Caroline Man-Yee Chan, Ci Chu, Michael Rossbach, Galih Kunarso, Tahira Bee Allapitchay, Kee Yew Wong, and Lawrence W. Stanton. 2008. "The Transcription Factor Zfp281 Controls Embryonic Stem Cell Pluripotency by Direct Activation and Repression of Target Genes." Stem Cells 26 (11): 2791-99.

Wickham, Hadley. 2016. ggplot2: Elegant Graphics for Data Analysis. Springer.

Wu, Hao, Ana C. D’Alessio, Shinsuke Ito, Kai Xia, Zhibin Wang, Kairong Cui, Keji Zhao, Yi Eve Sun, and Yi Zhang. 2011. "Dual Functions of Tet1 in Transcriptional Regulation in Mouse Embryonic Stem Cells." Nature 473 (7347): 389-93.

Wu, Qiang, Xi Chen, Jinqiu Zhang, Yuin-Han Loh, Teck-Yew Low, Weiwei Zhang, Wensheng Zhang, Siu-Kwan Sze, Bing Lim, and Huck-Hui Ng. 2006. "Sall4 Interacts with Nanog and Co-Occupies Nanog Genomic Sites in Embryonic Stem Cells." The Journal of Biological Chemistry 281 (34): 24090-94.

Xu, Huilei, Yen-Sin Ang, Ana Sevilla, Ihor R. Lemischka, and Avi Ma'ayan. 2014. "Construction and Validation of a Regulatory Network for Pluripotency and Self-Renewal of Mouse Embryonic Stem Cells.” PLoS Computational Biology 10 (8): e1003777.

$\mathrm{Xu}$, Huilei, Caroline Baroukh, Ruth Dannenfelser, Edward Y. Chen, Christopher M. Tan, Yan Kou, Yujin E. Kim, Ihor R. Lemischka, and Avi Ma'ayan. 2013. "ESCAPE: Database for Integrating High-Content Published Data Collected from Human and Mouse Embryonic Stem Cells." Database: The Journal of Biological Databases and Curation 2013 (June): bat045.

Yang, Peng, Yixuan Wang, Jiayu Chen, Hong Li, Lan Kang, Yu Zhang, She Chen, Bing Zhu, and Shaorong Gao. 2011. "RCOR2 Is a Subunit of the LSD1 Complex That Regulates ESC Property and Substitutes for SOX2 in Reprogramming Somatic Cells to Pluripotency." Stem Cells 29 (5): 791-801.

Yi, Fei, Laura Pereira, and Bradley James Merrill. 2008. "Tcf3 Functions as a Steady-State Limiter of Transcriptional Programs of Mouse Embryonic Stem Cell Self-Renewal." Stem Cells 26 (8): 1951-60.

Yu, J., M. A. Vodyanik, K. Smuga-Otto, J. Antosiewicz-Bourget, J. L. Frane, S. Tian, J. Nie, et al. 2007. "Induced Pluripotent Stem Cell Lines Derived from Human Somatic Cells." Science. https://doi.org/10.1126/science.1151526.

Zhang, Wensheng, Constantinos Chronis, Xi Chen, Heyao Zhang, Rapolas Spalinskas, Mercedes Pardo, Liangliang Chen, et al. 2019. "The BAF and PRC2 Complex Subunits Dpf2 and Eed Antagonistically Converge on Tbx3 to Control ESC Differentiation." Cell Stem Cell 24 (1): 138-52.e8.

Zhan, Ming, Daniel R. Riordon, Bin Yan, Yelena S. Tarasova, Sarah Bruweleit, Kirill V. Tarasov, Ronald A. Li, Robert P. Wersto, and Kenneth R. Boheler. 2012. "The B-MYB Transcriptional Network Guides Cell Cycle Progression and Fate Decisions to Sustain Self-Renewal and the Identity of Pluripotent Stem Cells." PloS One 7 (8): e42350.

Zhou, Yingyao, Bin Zhou, Lars Pache, Max Chang, Alireza Hadj Khodabakhshi, Olga Tanaseichuk, Christopher Benner, and Sumit K. Chanda. 2019. "Metascape Provides a Biologist-Oriented Resource for the Analysis of Systems-Level Datasets." Nature Communications 10 (1): 1523. 
Fiqu

A<smiles>[CH]1[CH]CC1</smiles>

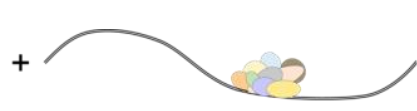

Transfect cells with 3xHA-tagged TALE

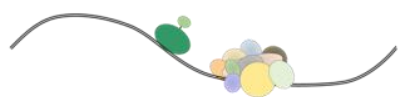

Fix cells, isolate and shear chromatin

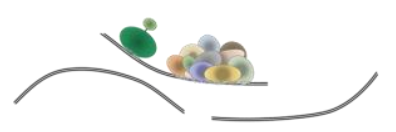

Purify target with Anti-HA agarose
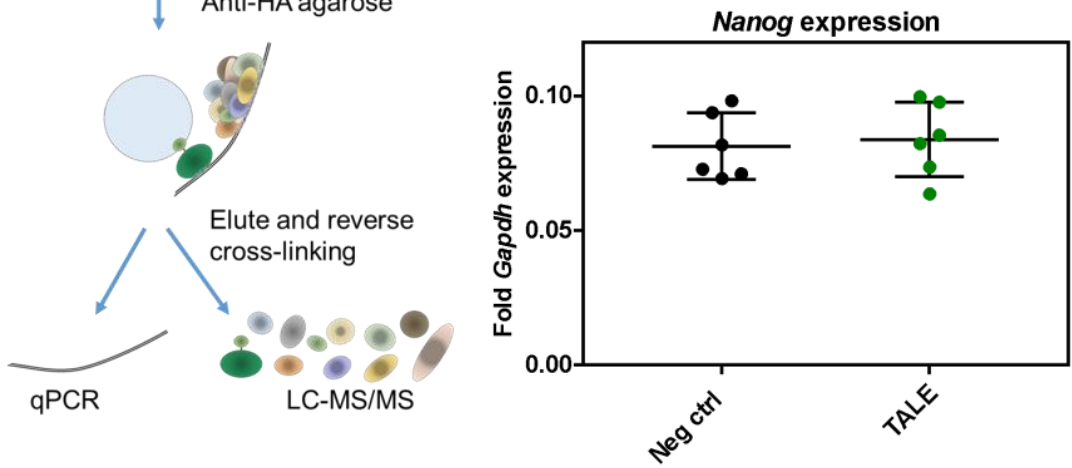

E

Replicate 1

Replicate 3

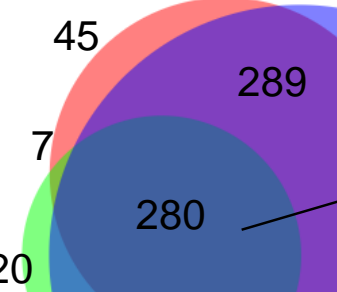

Replicate 2

84

604

$G$

Factor validation at the Nanog promoter

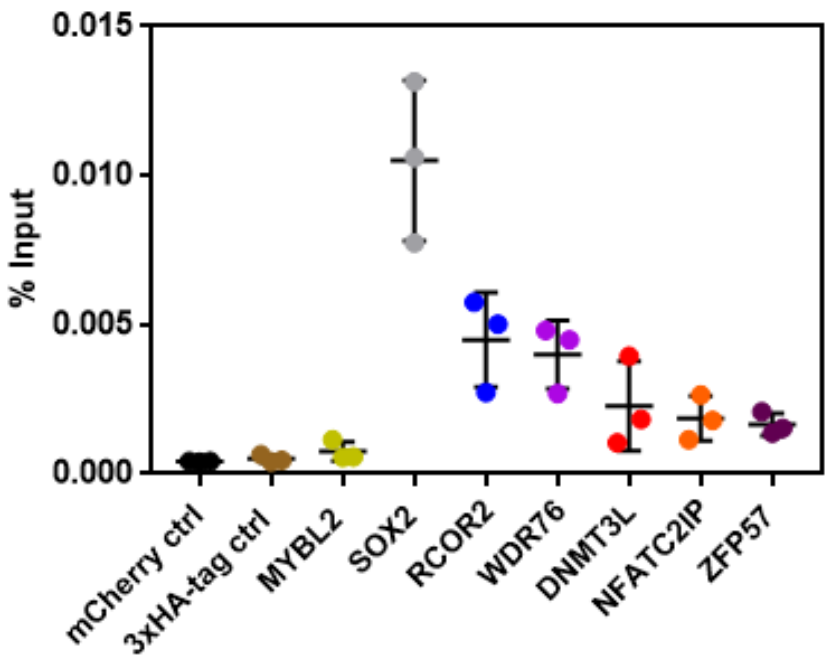

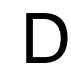

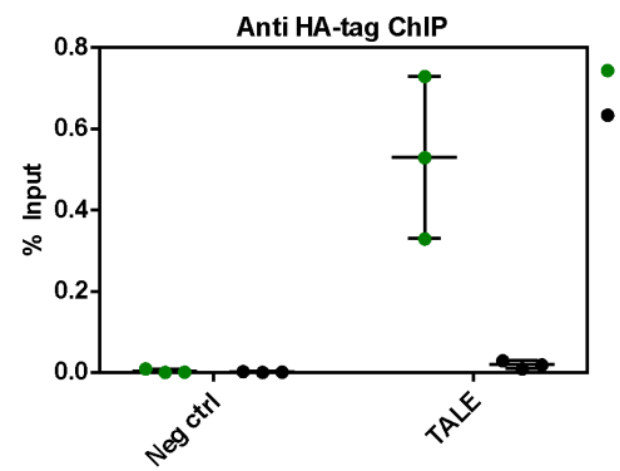

Nanog promoter

Background region

F

BRD4 (Liu et al. 2014)

DID01 (Liu et al. 2014)

DPF2 (Zhang et al. 2019)

ESRRB (Chen et al. 2008)

KLF4 (Chen et al. 2008 , Kim et al. 2008)

MYBL2 (B-MYB) (Zhan et al. 2012)

NANOG (Kim et al. 2008)

NR0B1 (DAX1) (Kim et al. 2008)

OCT4 (Chen et al. 2008 , Kim et al. 2008)

SOX2 (Chen et al. 2008, Kim et al. 2008)

TET1 (Wu et al. 2011)

TET2 (Sardina et al. 2018)

TFCP2L1 (TFCP2L1) (Chen et al. 2008)

ZFP281 (Wang et al. 2008)

F
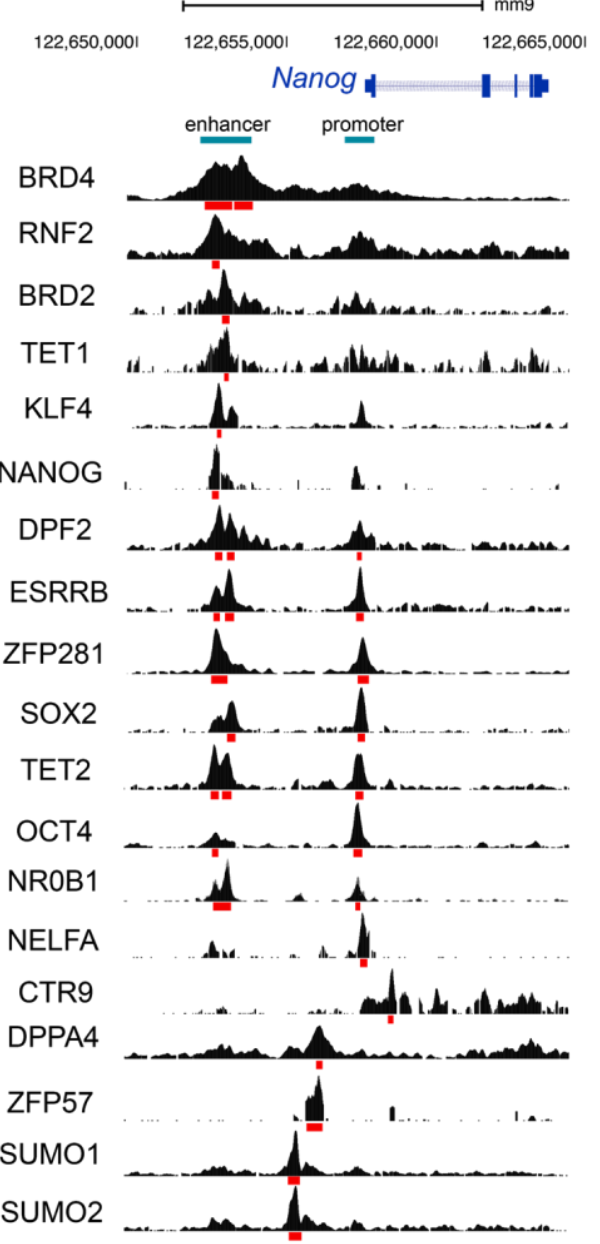
Figure 1. TINC allows isolation of the Nanog promoter complex from mouse ESCs.

(A) Schematic of the TINC method.

(B) A TALE was designed to bind to the proximal promoter of Nanog in mouse ESCs upstream of the OCT4 and SOX2 binding sites (Rodda et al. 2005). This region is located in open chromatin as shown by ATAC sequencing, which also shows the Nanog enhancer located approximately $5 \mathrm{~kb}$ upstream of the TSS and which is also bound by OCT4 and SOX2 (Knaupp et al. 2017). The TALE binding site is indicated in green, the OCT4 binding site in blue and the SOX2 binding site in gray.

(C) qPCR revealed that the expression of the TALE does not alter the expression of Nanog. Nanog expression levels were normalized to the housekeeping gene Gapdh.

(D) HA-tag ChIP-qPCR confirmed enrichment of the Nanog promoter only in TALE expressing cells and not in empty vector transfected control cells. This enrichment is specific to the Nanog promoter as a background genomic region (e.g. Sox2 regulatory region 2) was not enriched by the TALE.

(E) Overlap of the proteins identified in TINC replicates 1, 2 and 3. The 280 proteins identified by all three TINC replicates contain many of the previously published binders of the Nanog promoter and were hence used for further analysis.

(F) Validation of proteins identified by TINC utilizing publically available ChIP-seq data sets in the database ChIP-Atlas (Oki et al. 2018). Below each sample's BigWig track (black) are the respective peaks called by ChIP-Atlas (red).

(G) ChIP-qPCR of novel Nanog promoter binders identified by TINC. MYBL2 and SOX2 were used as positive controls. 
C.

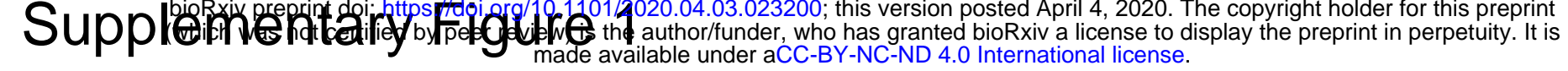

A
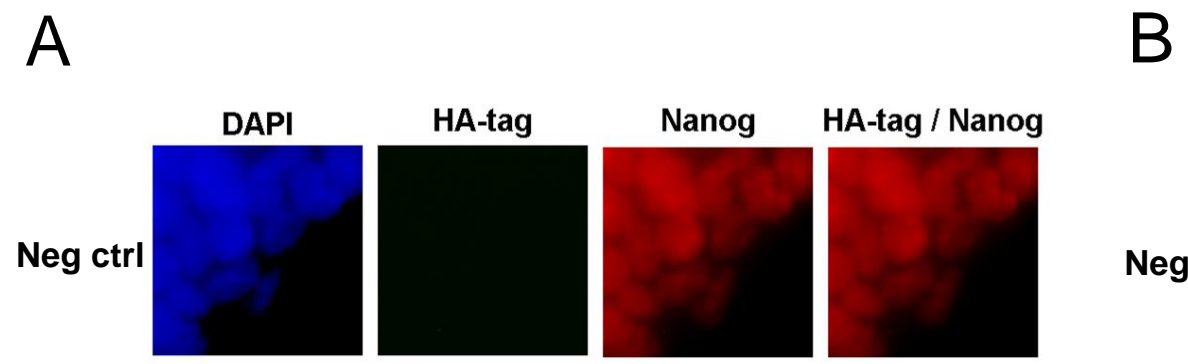

TALE
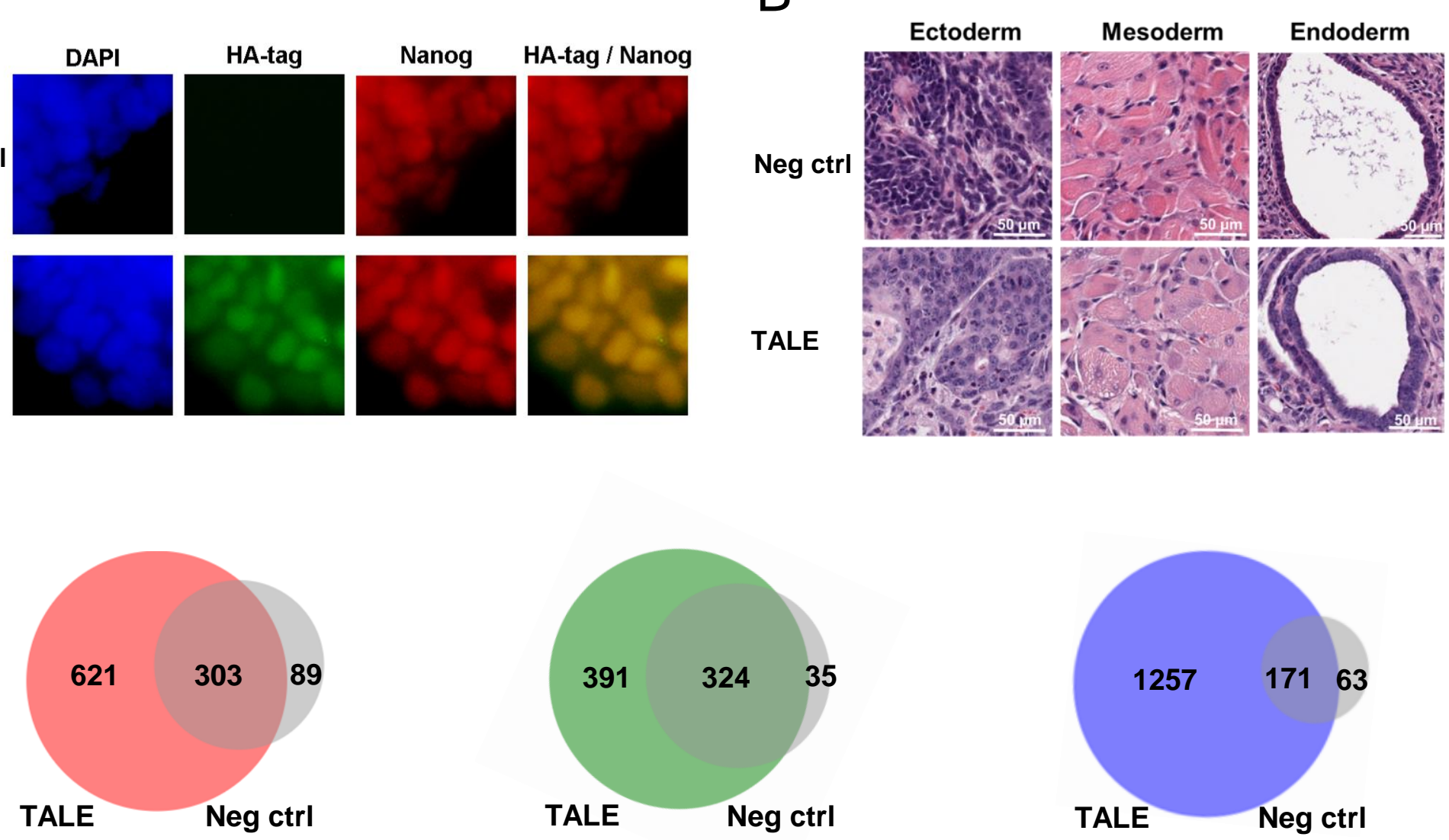

Replicate 1

Replicate 2

Replicate 3 


\section{Supplementary Figure 1.}

(A) Immunofluorescence shows homogenous expression of the TALE (e.g. anti HA-tag) and unaltered Nanog expression.

(B) Representative histology sections from teratomas derived from TALE-expressing ESCs and empty vector transfected control ESCs confirming the presence of derivatives of all three germ layers.

(C) Overlap of the proteins identified in each TALE replicate and the according negative control. Only proteins solely identified in the TALE sample but not the negative control were utilized for further analysis. 


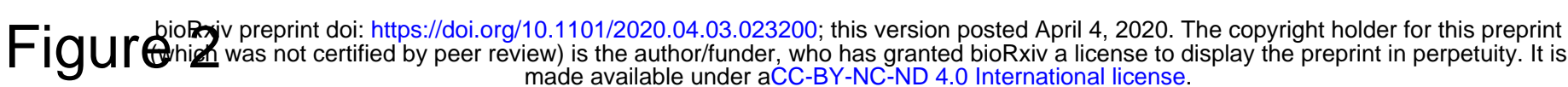

A

nucleic acid binding protein protein modifying enzyme

transcriptional reguecific chromatin/chromatin-binding or -regulatory protein chaperone

metabolite interconversion transporter

translational protein protein-binding
activity modulator extracellular matrix protein scaffold/adaptor protein intercellular signal molecule

$\begin{array}{cccc}0 & 20 & 40 & 60 \\ \text { number of proteins in category }\end{array}$

B

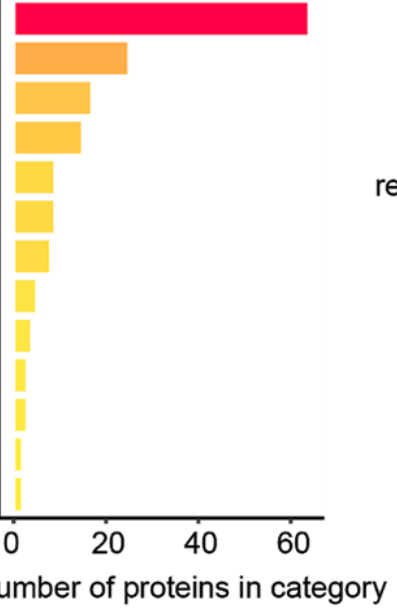

stem cell population maintenance Signaling pathways regulating pluripotency of stem cells response to leukemia

reproductive structure development regulation of intracellular steroid hormone receptor signaling pathway mitotic cell cycle process demethylation chromatin organization

Basal transcription factors ATP-dependent chromatin remodeling

Category $\bigcirc G O$ Biological Processes OKEGG Pathway

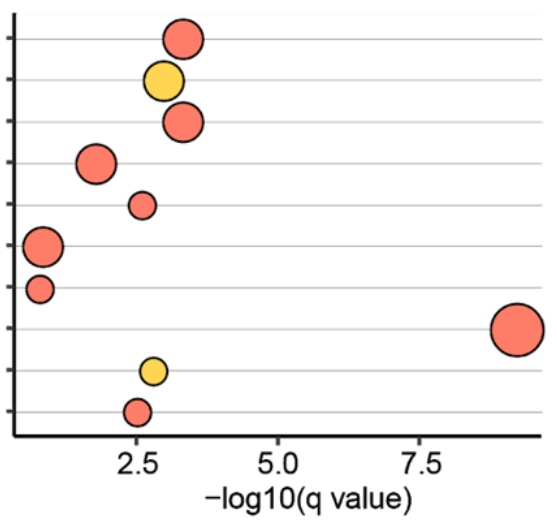

proteins $\circ \quad 0-5$ in term 0 06-10

○11-15

E

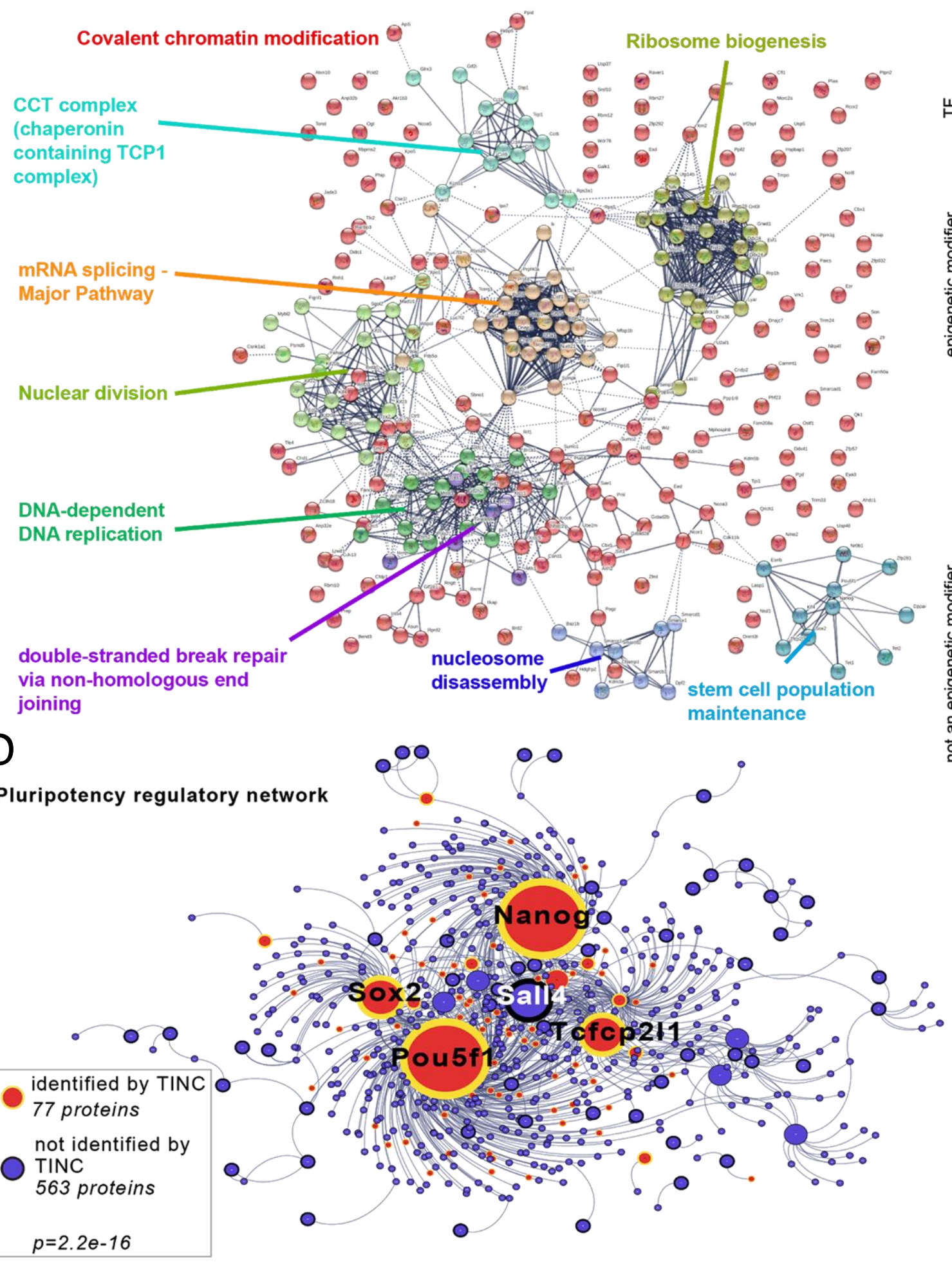

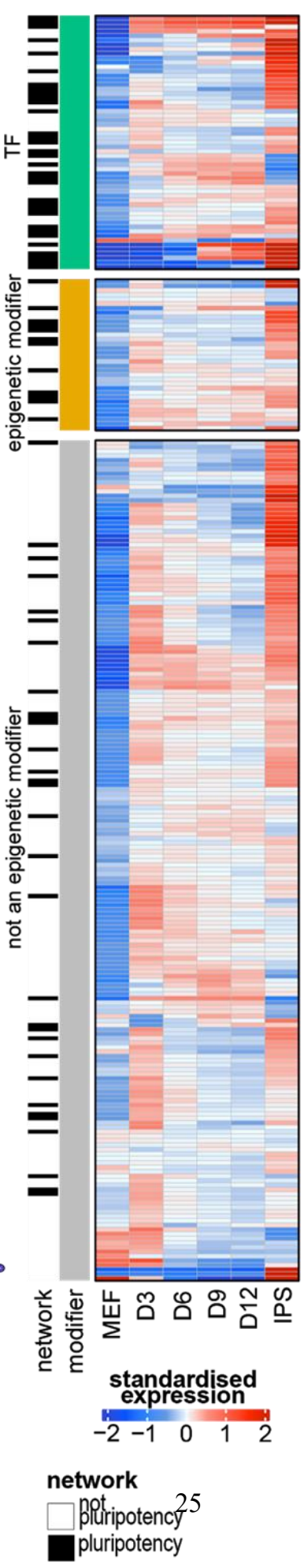




\section{Figure 2. Characterization of proteins interacting with the Nanog promoter.}

(A) Protein class categories for all 280 proteins identified by TINC. The color gradient corresponds to the number of proteins in the term with yellow being a few, and red being many. (B) GO Biological Process and KEGG pathway enrichment of transcription factors identified by TINC.

(C) Protein-protein interaction enrichment of the TINC proteins and k-means clustering analysis of the identified network by STRING as well as biological processes enriched for each of the clusters.

(D) The pluripotency regulatory network overlapped with proteins identified by TINC at the Nanog promoter. Significant enrichment of the TINC proteins in the pluripotency regulatory network was identified by a Fisher's exact test.

(E) Proteins identified by TINC with standardized expression values throughout the reprogramming process from MEFs to iPSCs. Proteins have been categorized into TFs, other epigenetic modifiers and all additional proteins. The black bars indicate whether they are a part of the pluripotency network from Figure 2D. 


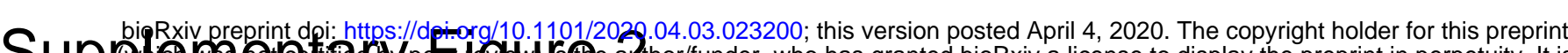
Supplemereitatary

A
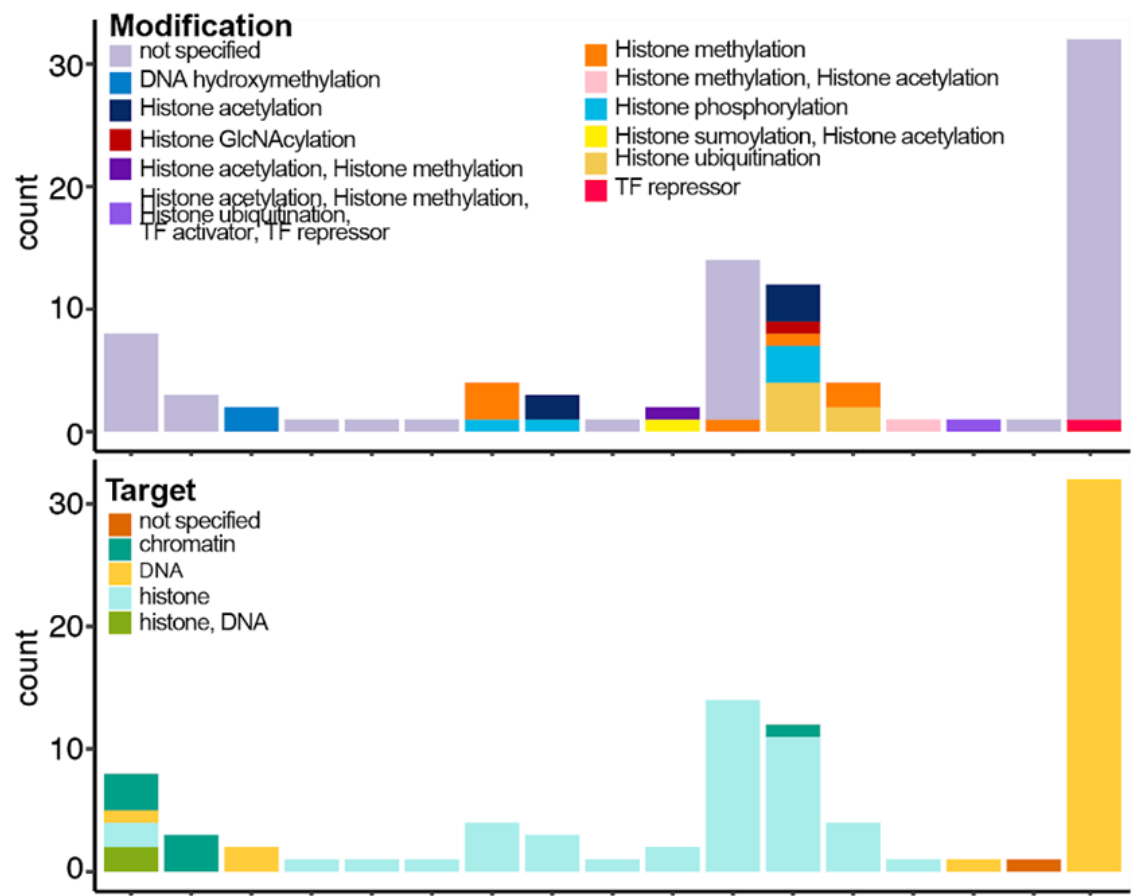

Function

D

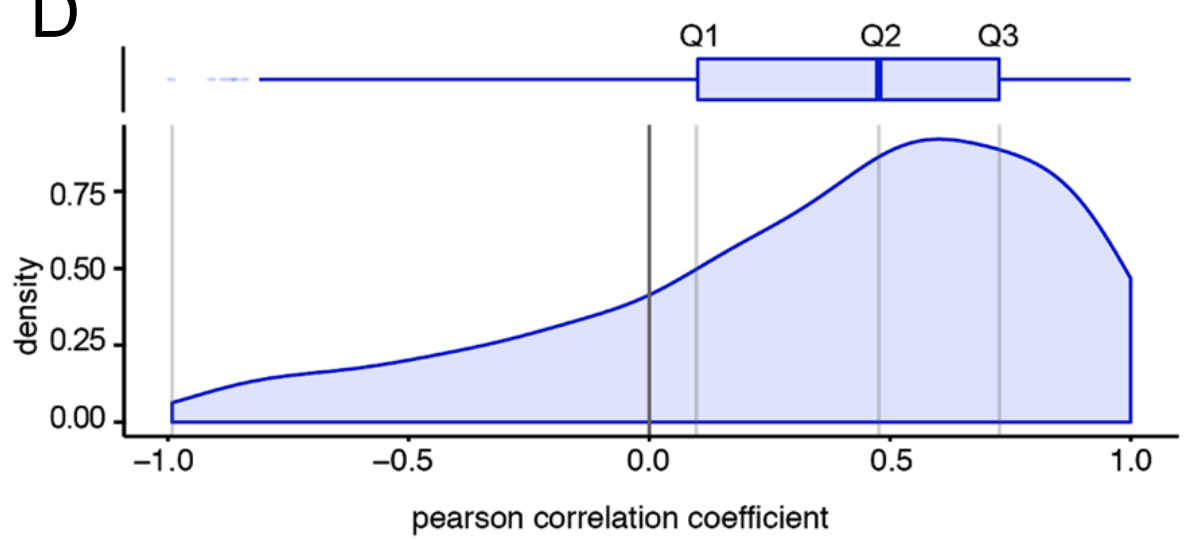

E negatively correlated genes to Nanog expression during reprogramming

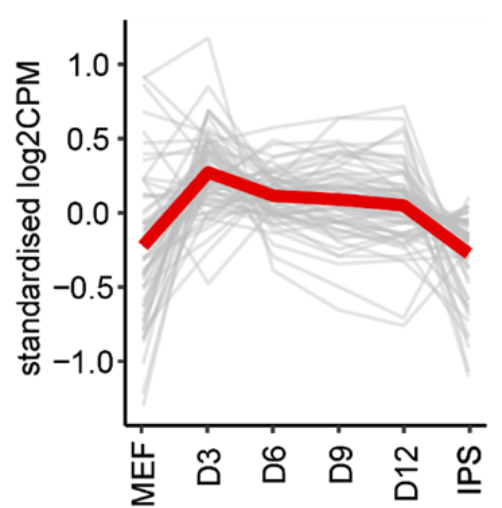

positively correlated genes to Nanog expression during reprogramming

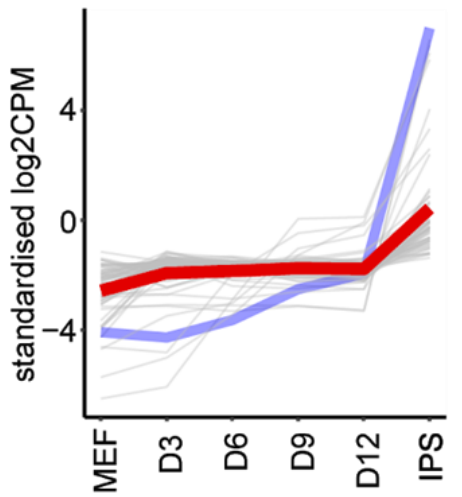

B

nucleic acid binding protein gene-specific transcriptional regulator chromatin chromatin-binding or -regulatory protein chaperone

protein modifying enzyme. transporter metabolite interconversion enzyme intercellular signal molecule
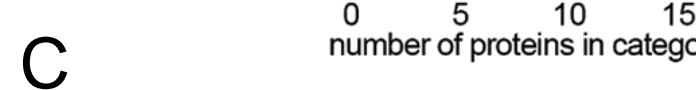
number of proteins in category

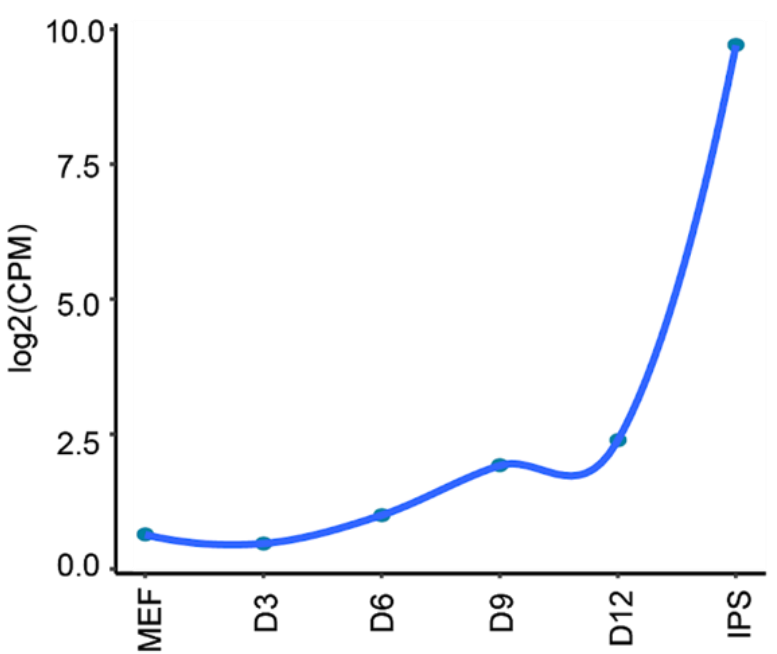

E regulation of DNA metabolic process

SUMOylation of DNA damage proteins protein modification by small protein conjugation or remova Generic Transcription Pathway dedifferentiation genetic imprinting regulation of chromatin silencing mitotic cell cycle process ribonucleoprotein complex biogenesis Cell Cycle Checkpoints mRNA processing

Protein ubiquitination Parvulin-associated pre-rRNP complex nucleosome disassembly protein import into nucleus mitotic metaphase plate congression nucleotide phosphorylation

DNA replication dephosphorylation

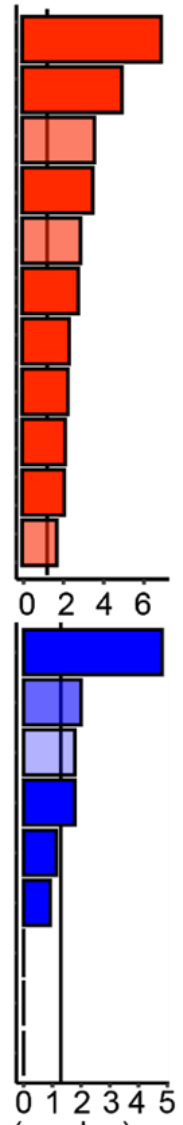

$-\log (q$ value) 


\section{Supplementary Figure 2.}

(A) The classification of all TFs and epigenetic modifiers identified by TINC at the Nanog promoter. The epigenetic factors were first categorized by their role or function and then further classified by the type of modification they exert as well as the type of molecules they target.

(B) Panther protein classification of the TINC proteins identified as part of the pluripotency regulatory network. The color gradient corresponds to the number of proteins in the term with yellow being a few and red being many.

(C) The log2CPM expression of Nanog throughout reprogramming.

(D) Distribution of Pearson's correlation coefficients of the expression of the candidates identified by TINC in relation to the expression of Nanog during reprogramming of MEFs to iPSCs.

(E) The standardized expression values of all negatively (62) and positively (218) correlated genes in comparison to Nanog during reprogramming of MEFs to iPSCs. Shown are also the mean trends of these TINC proteins during reprogramming as well as the expression of Nanog. (F) Gene ontology enrichment of biological processes and reactome gene sets of proteins identified by TINC at the Nanog promoter highly positively (top quantile, 70 genes) and negatively correlated to Nanog expression during reprogramming. 